\title{
Finite Population Mean Estimation through a Two-Parameter Ratio Estimator Using Auxiliary Information in Presence of Non-Response
}

\author{
S. K. PAL AND H. P. SINGH
}

\begin{abstract}
In surveys covering human populations it is observed that information in most cases are not obtained at the first attempt even after some callbacks. Such problems come under the category of non-response. Surveys suffer with non-response in various ways. It depends on the nature of required information, either surveys is concerned with general or sensitive issues of a society. Hansen and Hurwitz (1946) have considered the problem of non-response while estimating the population mean by taking a subsample from the non-respondent group with the help of extra efforts and an estimator was suggested by combining the information available from the response and nonresponse groups. We also mention that in survey sampling auxiliary information is commonly used to improve the performance of an estimator of a quantity of interest. For estimating the population mean using auxiliary information in presence of nonresponse has been discussed by various authors. In this paper, we have developed estimators for estimating the population mean of the variable under interest when there is non-response error in the study as well as in the auxiliary variable. We have studied properties of the suggested estimators under large sample approximation. Comparison of the suggested estimators with usual unbiased estimator reported by Hansen and Hurwitz (1946) and the ratio estimator due to Rao (1986) have been made. The results obtained are illustrated with aid of an empirical study.
\end{abstract}

Mathematics Subject Classification 2010: 62D05

Additional Key Words and Phrases: Study variable, Auxiliary variable, Non-response, Variance, Efficiency.

\section{INTRODUCTION}

In various human surveys, information is in most cases not obtained from all the units in the survey even after call backs. An estimate derived from such incomplete data may be misleading especially when the respondents differ from the non-respondents because the estimate can be biased. To cope with this problem, survey statisticians generally consider and adopt the non-respondents sub-sampling scheme developed by Hansen and Hurwitz (1946) to a wide range of practical situations. One topic which is discussed at great length in sampling theory is the 
estimator of population mean $\bar{Y}$ of the study variable $y$ using auxiliary information in presence of non-response. Cochran (1977) and Rao (1986) suggested the use of the ratio method of estimation for population mean $\bar{Y}$ of the study variable $y$ with sub-sampling from amongst the non- respondents.

When the population mean $\bar{X}$ of the auxiliary information $x$ is known, the work of Rao (1986) has been further extended by Khare and Srivastava (1997), Singh and Kumar (2008), Kumar (2012), Kumar and Vishwanathaiah (2013), Olufadi and Kumar (2014) and Chanu and Singh (2015) in presence of non-response.

For the case of non-response in sample survey, this paper addresses the problem of efficiently estimating the population mean $\bar{Y}$ of the study variable $y$ using auxiliary information. Taking motivation from Singh and Pal (2015) a two-parameter ratio estimators for population mean $\bar{Y}$ in presence of non-response using auxiliary variable $x$ have been proposed. The properties of these estimators have been studied in finite population approach under large sample approximation.

\section{THE USUAL RATIO AND PRODUCT ESTIMATORS}

Let $U=\left(U_{1}, U_{2}, \ldots, U_{N}\right)$ be a finite population of $N$ identifiable units and $(y, x)$ be the study and auxiliary variables respectively taking values $\left(y_{i}, x_{i}\right)$ on the $i^{\text {th }}$ population units $U_{i}, i=1,2, \ldots, . N$. Let $n$ be the size of a sample drown from the population of size $N$ by using simple random sampling without replacement (SRSWOR) to observe the study variable $y$. In this approach, the population of size $N$ is assumed to be composed of two strata of size $N_{1}$ and $N_{2}=\left(N-N_{1}\right)$ of 'respondent' and 'non-respondents' respectively. Out of $n$ units, $n_{1}$ respond and $n_{2}$ do not. From the $n_{2}$ non response units, $r\left(r=n_{2} / k, k>1\right)$ units are again randomly selected, hence of $n$ selected units we have $n_{1}+r$ observations on variable $y$. It is assumed that no non-response is observed in re-selected units.

Hansen and Hurwitz (1946) suggested the estimator of the population mean $\bar{Y}$ as 
$\bar{y}^{*}=\left(n_{1} / n\right) \bar{y}_{1}+\left(n_{1} / n\right) \bar{y}_{2}^{\prime}$,

where $\bar{y}_{1}=\sum_{i=1}^{n_{1}} y_{i} / n_{1}$ and $\bar{y}_{2}^{\prime}=\sum_{i=1}^{r} y_{i} / r$ are sample means based on $n_{1}$ and $r$ units respectively. The estimator $\bar{y}^{*}$ is unbiased estimator with variance given by

$$
\begin{aligned}
V\left(\bar{y}^{*}\right) & =\lambda S_{y}^{2}+\theta S_{y(2)}^{2} \\
& =\bar{Y}^{2}\left[\lambda C_{y}^{2}+\theta C_{y(2)}^{2}\right],
\end{aligned}
$$

where $f=n / N, \lambda=(1-f) / n, W_{2}=N_{2} / N ; \theta=W_{2}(k-1) / n$; and $S_{y}^{2}=\sum_{i=1}^{N}\left(y_{i}-\bar{Y}\right)^{2} /(N-1), S_{y_{(2)}}^{2}=\sum_{i=1}^{N_{2}}\left(y_{i}-\bar{Y}_{2}\right)^{2} /\left(N_{2}-1\right)$ and $\bar{Y}_{2}=\sum_{i=1}^{N_{2}} y_{i} / N_{2}$ are the population variances for the entire population, for the non-response group of the population, and the population mean of the non-response group respectively, $C_{y}^{2}=S_{y}^{2} / \bar{Y}^{2}$ and $C_{y(2)}^{2}=S_{y(2)}^{2} / \bar{Y}^{2}$.

When the population mean $\bar{X}$ of the auxiliary variable $x$ is known and information on $y$ and $x$ variables for the $n$ selected units is incomplete (designated as Case I) the usual ratio estimator for the population mean $\bar{Y}$ of the study variable $y$ is given by

$$
t_{R 1}=\left(\bar{y}^{*} / \bar{x}^{*}\right) \bar{X},
$$

where $\bar{x}^{*}=\left\{\left(n_{1} / n\right) \bar{x}_{1}+\left(n_{2} / n\right) \bar{x}_{2}^{\prime}\right\}$ is an unbiased estimator of the population mean $\bar{X}$ of the auxiliary variable $x, \bar{x}_{1}=\sum_{i=1}^{n_{1}}\left(x_{i} / n_{1}\right)$ and $\bar{x}_{2}^{\prime}=\sum_{i=1}^{r}\left(x_{i} / r\right)$.

The variance of the estimator $\bar{x}^{*}$ is given by

$$
\begin{aligned}
V\left(\bar{x}^{*}\right) & =\lambda S_{x}^{2}+\theta S_{x(2)}^{2} \\
& =\bar{X}^{2}\left[\lambda C_{x}^{2}+\theta C_{x(2)}^{2}\right],
\end{aligned}
$$

where $S_{x}^{2}$ and $S_{x(2)}^{2}$ are the respectively population variances for the whole population and for the non-response group, $C_{x}^{2}=S_{x}^{2} / \bar{X}^{2}$ and $C_{x(2)}^{2}=S_{x(2)}^{2} / \bar{X}^{2}$. 
If $\bar{X}$ is known and we have incomplete information on the study variable $y$ and the complete information on the auxiliary variable $x$ [designed as Case II], then an alternative ratio estimator is given by Rao (1986):

$t_{R 2}=\left(\bar{y}^{*} / \bar{x}\right) \bar{X}$,

where $\bar{x}=\sum_{i=1}^{n}\left(x_{i} / n\right)$ is the sample mean of the auxiliary variable $x$ based on a sample of size $n$. In similar fashion the conventional product estimation for the population mean $\bar{Y}$ of the study variable $y$ under Cases I and II are respectively defined by

$t_{P 1}=\bar{y}^{*}\left(\bar{x}^{*} / \bar{X}\right)$

and

$t_{P 2}=\bar{y}^{*}(\bar{x} / \bar{X})$

To the first degree of approximation, the mean squared errors of the ratio and product estimators are respectively given by

$$
\begin{aligned}
& \operatorname{MSE}\left(t_{R 1}\right)=\bar{Y}^{2}\left[\lambda\left(C_{y}^{2}+C_{x}^{2}-2 \rho_{y x} C_{y} C_{x}\right)+\theta\left(C_{y(2)}^{2}+C_{x(2)}^{2}-2 \rho_{y x(2)} C_{y(2)} C_{x(2)}\right)\right] \\
& \operatorname{MSE}\left(t_{R 2}\right)=\bar{Y}^{2}\left[\lambda\left(C_{y}^{2}+C_{x}^{2}-2 \rho_{y x} C_{y} C_{x}\right)+\theta C_{y(2)}^{2}\right], \\
& \operatorname{MSE}\left(t_{P 1}\right)=\bar{Y}^{2}\left[\lambda\left(C_{y}^{2}+C_{x}^{2}+2 \rho_{y x} C_{y} C_{x}\right)+\theta\left(C_{y(2)}^{2}+C_{x(2)}^{2}+2 \rho_{y x(2)} C_{y(2)} C_{x(2)}\right)\right],
\end{aligned}
$$

$$
\operatorname{MSE}\left(t_{P 2}\right)=\bar{Y}^{2}\left[\lambda\left(C_{y}^{2}+C_{x}^{2}+2 \rho_{y x} C_{y} C_{x}\right)+\theta C_{y(2)}^{2}\right]
$$

where $\rho_{y x}=\left(S_{y x} / S_{y} S_{x}\right)$ is correlation between $y$ and $x$ for the entire population, and $\rho_{y x(2)}=\left(S_{y x(2)} / S_{y(2)} S_{x(2)}\right)$ is the correlation coefficient between $y$ and $x$ for the 'non-respondent' group with

$$
S_{y x}=(N-1)^{-1} \sum_{i=1}^{N}\left(y_{i}-\bar{Y}\right)\left(x_{i}-\bar{X}\right) \text { and } S_{y x(2)}=\left(N_{2}-1\right)^{-1} \sum_{i=1}^{N_{2}}\left(y_{i}-\bar{Y}_{2}\right)\left(x_{i}-\bar{X}_{2}\right) \text {. }
$$

The ratio and product estimators $t_{R 1}$ and $t_{P 1}$ are better than the usual unbiased estimator $\bar{y}^{*}$ if 
(i) $C>(1 / 2)$ and $C_{(2)}>(1 / 2)$;

and

(ii) $C<-(1 / 2)$ and $C_{(2)}<-(1 / 2)$

respectively hold good,

where $C=\rho_{y x}\left(C_{y} / C_{x}\right)$ and $C_{(2)}=\rho_{y x(2)}\left(C_{y(2)} / C_{x(2)}\right)$.

We also note that the ratio estimator $t_{R 1}$ and the product estimator $t_{P 1}$ are also better than the usual unbiased estimator $\bar{y}^{*}$ respectively if

(iii) $R>(1 / 2)$,

(iv) $R<-(1 / 2)$,

where $R=\frac{\left(\lambda C C_{x}^{2}+\theta C_{(2)} C_{x(2)}^{2}\right)}{\left(\lambda C_{x}^{2}+\theta C_{x(2)}^{2}\right)}$.

The conditions (iii) and (iv) are not noticed in the literature.

Thus having the observations over the conditions (i) to (iv) the usual unbiased estimator $\bar{y}^{*}$ is to be preferred over $t_{R 1}$ and $t_{P 1}$ if the following conditions

(v) either $\left\{-(1 / 2) \leq C \leq(1 / 2)\right.$ and $\left.-(1 / 2) \leq C_{(2)} \leq(1 / 2)\right\}$

(vi) or $\quad\{-(1 / 2) \leq R \leq(1 / 2)\}$

holds true.

Further the estimators $t_{R 2}$ and $t_{P 2}$ are more efficient than the usual unbiased estimator $\bar{y}^{*}$ if

(i) $C>(1 / 2)$;

and

(ii) $C<-(1 / 2)$;

respectively hold true.

However, the usual unbiased estimator $\bar{y}^{*}$ is to be preferred over ratio estimator $t_{R 2}$ and product estimator $t_{P 2}$ if the condition:

$\{-(1 / 2) \leq C \leq(1 / 2)\}$ holds good. 
In this paper we have proposed a two-parameter ratio estimator for a finite population mean in the presence of non-response. We have obtained the bias and mean squared error (MSE) of the proposed class of estimators to the first degree of approximation. We have also derived the conditions for the parameter under which the proposed class of estimators has smaller MSE than the usual unbiased estimator $\bar{y}^{*}$, ratio estimator and product estimator. An empirical study is carried out in support of the present study.

\section{SOME SUGGESTED RATIO-TYPE ESTIMATORS}

In this section, we have suggested some ratio-type estimators for estimating the population mean $\bar{Y}$ in two different situations designated as Case I and Case II which are described below.

CASE I. When the population mean $\bar{X}$ of the auxiliary variable $x$ is known; and there is non-response on the study variable $y$ as well as on the auxiliary variable $x$. In this situation, we consider the following estimators for population mean $\bar{Y}$ as

$t_{1}^{*}=\bar{y}^{*}\left(\bar{X}^{2} / \bar{x}^{* 2}\right)$,

$t_{2}^{*}=\bar{y}^{*}\left(\bar{X} / \bar{x}^{*}\right)^{1 / 2}$,

$t_{3}^{*}=\bar{y}^{*} \exp \left\{\frac{\left(\bar{X}-\bar{x}^{*}\right)}{2\left(\bar{X}+\bar{x}^{*}\right)}\right\}$,

$t_{4}^{*}=\bar{y}^{*} \exp \left\{\frac{2\left(\bar{X}-\bar{x}^{*}\right)}{\left(\bar{X}+\bar{x}^{*}\right)}\right\}$,

$t_{5}^{*}=\bar{y}^{*}\left(\frac{\bar{X}}{\bar{x}^{*}}\right) \exp \left\{\frac{\left(\bar{X}-\bar{x}^{*}\right)}{\left(\bar{X}+\bar{x}^{*}\right)}\right\}$,

$t_{6}^{*}=\bar{y}^{*}\left(\frac{\bar{X}}{\bar{x}^{*}}\right)^{1 / 2} \exp \left\{\frac{\left(\bar{X}-\bar{x}^{*}\right)}{2\left(\bar{X}+\bar{x}^{*}\right)}\right\}$.

It is to be noted that estimators $t_{1}^{*}, t_{2}^{*}$ and $\left(t_{4}^{*}, t_{5}^{*}\right)$ are respectively defined on the lines of Kadilar and Cingi (2003), Swain (2014) and Singh and Pal (2015) respectively. 
The estimators in (3.1) to (3.6) are members of the following class of estimators of the population mean $\bar{Y}$ defined by

$t_{(\alpha, \delta)}=\bar{y}^{*}\left(\frac{\bar{X}}{\bar{x}^{*}}\right)^{\alpha} \exp \left\{\frac{\delta\left(\bar{X}-\bar{x}^{*}\right)}{\left(\bar{X}+\bar{x}^{*}\right)}\right\}$,

where $(\alpha, \delta)$ are suitable chosen constants. We note that the class of estimators:

(i) $\quad t_{(\alpha, \delta)} \rightarrow t_{1}^{*}$ for $(\alpha, \delta)=(2,0)$,

(ii) $\quad t_{(\alpha, \delta)} \rightarrow t_{2}^{*}$ for $(\alpha, \delta)=\left(\frac{1}{2}, 0\right)$,

(iii) $\quad t_{(\alpha, \delta)} \rightarrow t_{3}^{*}$ for $(\alpha, \delta)=\left(0, \frac{1}{2}\right)$,

(iv) $\quad t_{(\alpha, \delta)} \rightarrow t_{4}^{*}$ for $(\alpha, \delta)=(0,2)$,

(v) $\quad t_{(\alpha, \delta)} \rightarrow t_{5}^{*}$ for $(\alpha, \delta)=(1,1)$,

(vi) $\quad t_{(\alpha, \delta)} \rightarrow t_{6}^{*}$ for $(\alpha, \delta)=\left(\frac{1}{2}, \frac{1}{2}\right)$.

In addition to $t_{1}^{*}$ to $t_{6}^{*}$, many other acceptable estimators can be generated from the class of estimators $t_{(\alpha, \delta)}$. Thus to obtain the biases and MSEs of the estimators $t_{1}^{*}$ to $t_{6}^{*}$, we will first obtained the bias and $M S E$ of the generalized class of estimators $t_{(\alpha, \delta)}$.

To obtain the bias and MSE of the class of estimators $t_{(\alpha, \delta)}$, we write $\bar{y}^{*}=\bar{Y}\left(1+e_{0}\right), \bar{x}^{*}=\bar{X}\left(1+e_{1}\right)$

such that

$E\left(e_{0}\right)=E\left(e_{1}\right)=0$

and

$E\left(e_{0}^{2}\right)=\left(\lambda C_{y}^{2}+\theta C_{y(2)}^{2}\right), E\left(e_{1}^{2}\right)=\left(\lambda C_{x}^{2}+\theta C_{x(2)}^{2}\right), E\left(e_{0} e_{1}\right)=\left(\lambda C C_{x}^{2}+\theta C_{(2)} C_{x(2)}^{2}\right)$.

Now expressing (3.7) in terms of e's we have 


$$
\begin{aligned}
t_{(\alpha, \delta)} & =\bar{Y}\left(1+e_{0}\right)\left(1+e_{1}\right)^{-\alpha} \exp \left\{\frac{-\delta e_{1}}{\left(2+e_{1}\right)}\right\} \\
& =\bar{Y}\left(1+e_{0}\right)\left(1+e_{1}\right)^{-\alpha} \exp \left\{\frac{-\delta e_{1}}{2}\left(1+\frac{e_{1}}{2}\right)^{-1}\right\} .
\end{aligned}
$$

We assume that $\left|e_{1}\right|<1$ so that $\left(1+e_{1}\right)^{-\alpha}$ and $\left(1+\frac{e_{1}}{2}\right)^{-1}$ are expandable in terms of power series. Expanding and multiplying out the right hand side of (3.8) and neglecting terms of e's having power greater than two we have

$$
t_{(\alpha, \delta)} \cong \bar{Y}\left[1+e_{0}-\frac{(\delta+2 \alpha)}{2} e_{1}-\frac{(\delta+2 \alpha)}{2} e_{0} e_{1}+\frac{(\delta+2 \alpha)(\delta+2 \alpha+2)}{8} e_{1}^{2}\right]
$$

or

$$
\left(t_{(\alpha, \delta)}-\bar{Y}\right) \cong \bar{Y}\left[e_{0}-\frac{(\delta+2 \alpha)}{2} e_{1}-\frac{(\delta+2 \alpha)}{2} e_{0} e_{1}+\frac{(\delta+2 \alpha)(\delta+2 \alpha+2)}{8} e_{1}^{2}\right]
$$

Taking expectation of both sides of (3.9) we get the bias of the class of estimators $t_{(\alpha, \delta)}$ to the first degree of approximation as

$$
B\left(t_{(\alpha, \delta)}\right)=\frac{\bar{Y}(\delta+2 \alpha)}{8}\left(\lambda C_{x}^{2}+\theta C_{x(2)}^{2}\right)(\delta+2 \alpha-4 R+2)
$$

which is zero, if either

$$
\delta=-2 \alpha(\text { or } \alpha=-\delta / 2)
$$

or

$$
\delta=-2(\alpha-2 R+1)(\text { or } \alpha=2 R-(\delta / 2)-1) .
$$

The suggested class of estimators $t_{(\alpha, \delta)}$ substituted with the values of $\delta$ (or $\alpha$ ) from (3.11) and (3.12) becomes an (approximately) unbiased estimator for the population mean $\bar{Y}$ respectively as

$$
\begin{aligned}
t_{(\alpha,-2 \alpha)} & =\bar{y}^{*}\left(\frac{\bar{X}}{\bar{x}^{*}}\right)^{\alpha} \exp \left\{\frac{2 \alpha\left(\bar{x}^{*}-\bar{X}\right)}{\left(\bar{X}+\bar{x}^{*}\right)}\right\} \\
& =t_{u(\alpha)}(\text { say })
\end{aligned}
$$


and

$$
\begin{aligned}
t_{(\delta / 2, \delta)} & =\bar{y}^{*}\left(\frac{\bar{x}^{*}}{\bar{X}}\right)^{\delta / 2} \exp \left\{\frac{\delta\left(\bar{X}-\bar{x}^{*}\right)}{\left(\bar{X}+\bar{x}^{*}\right)}\right\} \\
& =t_{u(\delta)}(\text { say })
\end{aligned}
$$

Here we note that the estimators $t_{u(\alpha)}$ and $t_{u(\delta)}$ are almost unbiased irrespective of the values of $(\alpha, \delta)$.

Squaring both sides of (3.9) and neglecting terms of e's having power greater than two we have

$$
\left(t_{(\alpha, \delta)} \bar{Y}\right)^{2} \cong \bar{Y}^{2}\left[e_{0}^{2}+\frac{(\delta+2 \alpha)^{2}}{4} e_{1}^{2}-(\delta+2 \alpha) e_{0} e_{1}\right]
$$

The mean squared error of the proposed class of estimators $t_{(\alpha, \delta)}$ to the first degree approximation is given by

$$
\operatorname{MSE}\left(t_{(\alpha, \delta)}\right)=\bar{Y}^{2}\left[\left(\lambda C_{y}^{2}+\theta C_{y(2)}^{2}\right)+\left(\lambda C_{x}^{2}+\theta C_{x(2)}^{2}\right)\left\{\frac{(\delta+2 \alpha)^{2}}{4}-(\delta+2 \alpha) R\right\}\right]
$$

which is minimum when

$$
(\delta+2 \alpha)=2 R \text {. }
$$

Putting (3.17) in (3.16) we get the minimum MSE of $t_{(\alpha, \delta)}$ as

$$
\min \cdot \operatorname{MSE}\left(t_{(\alpha, \delta)}\right)=\bar{Y}^{2}\left[\left(\lambda C_{y}^{2}+\theta C_{y(2)}^{2}\right)-\frac{\left(\lambda C C_{x}^{2}+\theta C_{(2)} C_{x(2)}^{2}\right)^{2}}{\left(\lambda C_{x}^{2}+\theta C_{x(2)}^{2}\right)}\right]
$$

Thus we state that the following theorem.

THEOREM 3.1. To the first degree of approximation,

$$
\operatorname{MSE}\left(t_{(\alpha, \delta)}\right) \geq \bar{Y}^{2}\left(\lambda C_{y}^{2}+\theta C_{y(2)}^{2}\right)\left(1-\rho^{* 2}\right)
$$

with equality holding if

$(\delta+2 \alpha)=2 R$, 
where $\rho^{*}=\frac{\operatorname{Cov}\left(\bar{y}^{*}, \bar{x}^{*}\right)}{\sqrt{V\left(\bar{y}^{*}\right) V\left(\bar{x}^{*}\right)}}=\frac{\left(\lambda C C_{x}^{2}+\theta C_{(2)} C_{x(2)}^{2}\right)}{\sqrt{\left(\lambda C_{y}^{2}+\theta C_{y(2)}^{2}\right)\left(\lambda C_{x}^{2}+\theta C_{x(2)}^{2}\right)}}$.

Noting from Srivastava $(1971,1980)$ it can be shown that the minimum mean squared error of the class of estimators $t_{(\alpha, \delta)}$ in (3.18) is the minimal possible mean squared error up to first degree of approximation for a large class of estimators to which the estimators $t_{i}^{*}(i=1$ to 6$)$ and the class of estimators $t_{(\alpha, \delta)}$ also belong, for example, for the estimators of the form:

$$
t_{g}=\bar{y}^{*} g\left(\bar{x}^{*} / \bar{X}\right)
$$

where $g(\bullet)$ is a function of $\left(\bar{x}^{*} / \bar{X}\right)$ with $g(1)=1$.

\section{COMPARISON OF THE PROPOSED CLASS OF ESTIMATORS $t_{(\alpha, \delta)}$ WITH HANSEN AND HURWITZ (1946) ESTIMATOR $\bar{y}^{*}$, RAO (1986) RATIO ESTIMATOR $t_{R 1}$ AND PRODUCT ESTIMATOR $t_{P 1}$}

From (2.2) and (3.16) we have

$$
\begin{gathered}
\operatorname{Var}\left(\bar{y}^{*}\right)-\operatorname{MSE}\left(t_{(\alpha, \delta)}\right)=\bar{Y}^{2}(\delta+2 \alpha)\left[\left(\lambda C C_{x}^{2}+\theta C_{(2)} C_{x(2)}^{2}\right)-\frac{(\delta+2 \alpha)\left(\lambda C_{x}^{2}+\theta C_{x(2)}^{2}\right)}{4}\right] \\
=\bar{Y}^{2}(\delta+2 \alpha)\left[\lambda C_{x}^{2}\left\{C-\frac{(\delta+2 \alpha)}{4}\right\}+\theta C_{x(2)}^{2}\left\{C_{(2)}-\frac{(\delta+2 \alpha)}{4}\right\}\right]
\end{gathered}
$$

which is positive if

$$
\left.\begin{array}{l}
\text { either } C>\frac{(\delta+2 \alpha)}{4} \text { and } C_{(2)}>\frac{(\delta+2 \alpha)}{4} \text { with }(\delta+2 \alpha)>0 \\
\text { or } \quad C<\frac{(\delta+2 \alpha)}{4} \text { and } C_{(2)}<\frac{(\delta+2 \alpha)}{4} \text { with }(\delta+2 \alpha)<0
\end{array}\right\} \text {. }
$$

The expression in (4.1) can be re-expressed as

$$
\operatorname{Var}\left(\bar{y}^{*}\right)-\operatorname{MSE}\left(t_{(\alpha, \delta)}\right)=\bar{Y}^{2}(\delta+2 \alpha)\left(\lambda C_{x}^{2}+\theta C_{x(2)}^{2}\right)\left[R-\frac{(\delta+2 \alpha)}{4}\right]
$$

which is positive if 
either $R>\frac{(\delta+2 \alpha)}{4}$ with $\left.(\delta+2 \alpha)>0\right)$.

or $\left.\quad R<\frac{(\delta+2 \alpha)}{4} \operatorname{with}(\delta+2 \alpha)<0\right\}$

Thus the proposed class of estimators $t_{(\alpha, \delta)}$ is more efficient than usual unbiased estimator $\bar{y}^{*}$ if either the condition in (4.2) or the condition (4.4) holds good. However, the condition (4.2) is sufficient for the proposed class of estimators $t_{(\alpha, \delta)}$ to be better than the usual unbiased estimator $\bar{y}^{*}$.

From (2.8) and (3.16) we have

$$
\begin{aligned}
\operatorname{MSE}\left(t_{R 1}\right)-\operatorname{MSE}\left(t_{(\alpha, \delta)}\right) & =\bar{Y}^{2}\left\{1-\frac{(\delta+2 \alpha)}{2}\right\}\left[\lambda C_{x}^{2}\left(1+\frac{(\delta+2 \alpha)}{2}-2 C\right)\right. \\
& \left.+\theta C_{x(2)}^{2}\left(1+\frac{(\delta+2 \alpha)}{2}-2 C_{(2)}\right)\right]
\end{aligned}
$$

which is positive if

$$
\left.\begin{array}{l}
\text { either } C>\frac{(\delta+2 \alpha+2)}{4} \text { and } C_{(2)}>\frac{(\delta+2 \alpha+2)}{4} \text { with } \frac{(\delta+2 \alpha)}{2}>1 \\
\text { or } \quad C<\frac{(\delta+2 \alpha+2)}{4} \text { and } C_{(2)}<\frac{(\delta+2 \alpha+2)}{4} \text { with } \frac{(\delta+2 \alpha)}{2}<1
\end{array}\right\}
$$

Expression (4.5) can also be written as

$$
\operatorname{MSE}\left(t_{R 1}\right)-\operatorname{MSE}\left(t_{(\alpha, \delta)}\right)=\bar{Y}^{2}\left(\lambda C_{x}^{2}+\theta C_{x(2)}^{2}\right)\left\{1-\frac{(\delta+2 \alpha)}{2}\right\}\left(\frac{(\delta+2 \alpha+2)}{2}-2 R\right)
$$

which is positive if

$$
\left.\begin{array}{l}
\text { either } \quad R>\frac{(\delta+2 \alpha+1)}{2} \text { with } \frac{(\delta+2 \alpha)}{2}>1 \\
\text { or } \quad R<\frac{(\delta+2 \alpha+1)}{2} \text { with } \frac{(\delta+2 \alpha)}{2}<1
\end{array}\right\}
$$

Thus the proposed class of estimators $t_{(\alpha, \delta)}$ is better than the usual unbiased estimator $t_{R 1}$ if either the condition in (4.6) or the condition in (4.8) holds good. However, the condition in (4.6) is sufficient for the proposed class of estimators 
$t_{(\alpha, \delta)}$ to be better than ratio estimator $t_{R 1}$.

From (2.10) and (3.16) we have

$$
\begin{aligned}
\operatorname{MSE}\left(t_{P 1}\right)-\operatorname{MSE}\left(t_{(\alpha, \delta)}\right) & =\bar{Y}^{2}\left\{1+\frac{(\delta+2 \alpha)}{2}\right\}\left[\lambda C_{x}^{2}\left(2 C-\frac{(\delta+2 \alpha)}{2}+1\right)\right. \\
& \left.+\theta C_{x(2)}^{2}\left(2 C_{(2)}-\frac{(\delta+2 \alpha)}{2}+1\right)\right]
\end{aligned}
$$

which is non-negative if

$$
\left.\begin{array}{l}
\text { either } C>-\frac{1}{2}+\frac{(\delta+2 \alpha)}{4} \text { and } C_{(2)}>-\frac{1}{2}+\frac{(\delta+2 \alpha)}{4} \text { with } \frac{(\delta+2 \alpha)}{2}>-1 \\
\text { or } \quad C<-\frac{1}{2}+\frac{(\delta+2 \alpha)}{4} \text { and } C_{(2)}<-\frac{1}{2}+\frac{(\delta+2 \alpha)}{4} \text { with } \frac{(\delta+2 \alpha)}{2}<-1
\end{array}\right\} \text {. }
$$

Expression (4.9) be can also written as

$$
\operatorname{MSE}\left(t_{P 1}\right)-\operatorname{MSE}\left(t_{(\alpha, \delta)}\right)=\bar{Y}^{2}\left(\lambda C_{x}^{2}+\theta C_{x(2)}^{2}\right)\left\{1+\frac{(\delta+2 \alpha)}{2}\right\}\left(2 R-\frac{(\delta+2 \alpha)}{2}+1\right)
$$

which is positive if

$$
\left.\begin{array}{ll}
\text { either } & R>-\frac{1}{2}+\frac{(\delta+2 \alpha)}{4} \text { with } \frac{(\delta+2 \alpha)}{2}>-1 \\
\text { or } \quad & R<-\frac{1}{2}+\frac{(\delta+2 \alpha)}{4} \text { with } \frac{(\delta+2 \alpha)}{2}<-1
\end{array}\right\} \text {. }
$$

Thus the proposed class of estimators $t_{(\alpha, \delta)}$ is more efficient than product estimator $t_{P 1}$ if the condition in (4.10) or the condition (4.12) is satisfied. However, the condition (4.10) is sufficient for the proposed class of estimators $t_{(\alpha, \delta)}$ to be better than the product estimator $t_{P 1}$. 
4.1. Mean Squared Errors of the Estimators $t_{i}^{*}(i=1$ to 6$)$

Putting $(\alpha, \delta)=(2,0),\left(\frac{1}{2}, 0\right),\left(0, \frac{1}{2}\right),(0,2),(1,1),\left(\frac{1}{2}, \frac{1}{2}\right)$ in $(3.16)$ we get the MSEs of the estimators $t_{i}^{*}(i=1$ to 6$)$ to the first degree of approximation as

$$
\begin{aligned}
& \operatorname{MSE}\left(t_{1}^{*}\right)=\bar{Y}^{2}\left[\left(\lambda C_{y}^{2}+\theta C_{y(2)}^{2}\right)+4\left(\lambda C_{x}^{2}+\theta C_{x(2)}^{2}\right)(1-R)\right], \\
& \operatorname{MSE}\left(t_{2}^{*}\right)=\bar{Y}^{2}\left[\left(\lambda C_{y}^{2}+\theta C_{y(2)}^{2}\right)+\left(\lambda C_{x}^{2}+\theta C_{x(2)}^{2}\right)\left(\frac{1}{4}-R\right)\right] \\
& \operatorname{MSE}\left(t_{3}^{*}\right)=\bar{Y}^{2}\left[\left(\lambda C_{y}^{2}+\theta C_{y(2)}^{2}\right)+\frac{1}{16}\left(\lambda C_{x}^{2}+\theta C_{x(2)}^{2}\right)(1-8 R)\right], \\
& \operatorname{MSE}\left(t_{4}^{*}\right)=\bar{Y}^{2}\left[\left(\lambda C_{y}^{2}+\theta C_{y(2)}^{2}\right)+\left(\lambda C_{x}^{2}+\theta C_{x(2)}^{2}\right)(1-2 R)\right], \\
& \operatorname{MSE}\left(t_{5}^{*}\right)=\bar{Y}^{2}\left[\left(\lambda C_{y}^{2}+\theta C_{y(2)}^{2}\right)+3\left(\lambda C_{x}^{2}+\theta C_{x(2)}^{2}\right)\left(\frac{3}{4}-R\right)\right], \\
& \operatorname{MSE}\left(t_{6}^{*}\right)=\bar{Y}^{2}\left[\left(\lambda C_{y}^{2}+\theta C_{y(2)}^{2}\right)+\frac{3}{2}\left(\lambda C_{x}^{2}+\theta C_{x(2)}^{2}\right)\left(\frac{3}{8}-R\right)\right] .
\end{aligned}
$$

The estimators $t_{1}^{*}, t_{2}^{*}, t_{3}^{*}, t_{4}^{*}, t_{5}^{*}$ and $t_{6}^{*}$ are respectively better than $\bar{y}^{*}$ if

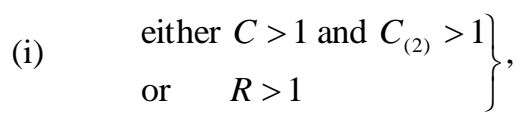

(ii) either $C>\frac{1}{4}$ and $C_{(2)}>\frac{1}{4}$,

$$
\text { or } \quad R>\frac{1}{4}
$$

(iii) either $C>\frac{1}{8}$ and $C_{(2)}>\frac{1}{8}$, or $\left.R>\frac{1}{8}\right\}$

(iv) or $\quad R>\frac{1}{2}$ 
(v) either $C>\frac{3}{4}$ and $\left.C_{(2)}>\frac{3}{4}\right\}$, or $\quad R>\frac{3}{4}$

(vi) either $C>\frac{3}{8}$ and $C_{(2)}>\frac{3}{8}$. or $\quad R>\frac{3}{8}$

The estimators $t_{1}^{*}, t_{2}^{*}, t_{3}^{*}, t_{4}^{*}, t_{5}^{*}$ and $t_{6}^{*}$ are respectively more efficient than the ratio estimator $t_{R 1}$ if

(i)

$$
\left.\begin{array}{l}
\text { either } C>\frac{3}{2} \text { and } C_{(2)}>\frac{3}{2} \\
\text { or } \quad R>\frac{3}{2}
\end{array}\right\} \text {, }
$$

$\left.\begin{array}{rl}\text { (ii) } \quad \text { either } C & >\frac{3}{4} \text { and } C_{(2)}>\frac{3}{4} \\ \text { or } \quad & R>1\end{array}\right\}$,

(iii) either $C>\frac{5}{8}$ and $\left.C_{(2)}>\frac{5}{8}\right\}$, or $\quad R>\frac{3}{4}$

(iv)

$\left.\begin{array}{l}\text { either } C>1 \text { and } C_{(2)}>1 \\ \text { or } \quad R>\frac{3}{2}\end{array}\right\}$,

(v) $\left.\begin{array}{rl}\text { either } C & >\frac{5}{4} \text { and } C_{(2)}>\frac{5}{4} \\ \text { or } \quad R & >2\end{array}\right\}$,

(vi) either $C>\frac{7}{8}$ and $C_{(2)}>\frac{7}{8}$ or $\quad R>\frac{5}{4}$

It is observed from (4.19) to (4.24) and (4.25) to (4.30) that the proposed estimator $t_{1}^{*}, t_{2}^{*}, t_{3}^{*}, t_{4}^{*}, t_{5}^{*}$ and $t_{6}^{*}$ are more efficient than the usual unbiased estimator 
$\bar{y}^{*}$ and the ratio estimator $t_{R 1}$ as long as the corresponding conditions given by (4.25) to (4.30) are satisfied.

4.2. Mean Squared Errors of the Almost unbiased Estimators $t_{u(\alpha)}$ and $t_{u(\delta)}$

Inserting $\delta=-2 \alpha$ and $\alpha=-(\delta / 2)$ in (3.16) we get the mean squared error of $t_{u(\alpha)}$ and $t_{u(\delta)}$ to the first degree of approximation as

$\operatorname{MSE}\left(t_{u(\alpha)}\right)=\operatorname{MSE}\left(t_{u(\delta)}\right)=\bar{Y}^{2}\left(\lambda C_{y}^{2}+\theta C_{y(2)}^{2}\right)$

which equals to the variance of usual unbiased estimator $\bar{y}^{*}$.

REMARK 4.2.1. Following the procedure adopting in Rao (1983), the cost aspects can be easily discussed when there is non-response on both the variables $y$ and $x$.

REMARK 4.2.2. One can also consider the proposed estimator for the population mean under double (or two phase) sampling in presence of non-response where the population mean $\bar{X}$ of the auxiliary variable $x$ is not known. For the estimate of mean $\bar{X}$ of the auxiliary variable $x$, a large first phase sample of size $n^{\prime}$ is selected from a population of $N$ units by simple random sampling without replacement (SRSWOR). A smaller second phase sample of size $n$ is selected from $n^{\prime}$ by SRSWOR sampling scheme and the study variable $y$ is measured on it.

Thus the double sampling version of the proposed estimator $t_{(\alpha, \delta)}$ at (3.7) in presence of non-response on both the variables $y$ and $x$, is given by

$$
t_{(\alpha, \delta)}^{(d)}=\bar{y}^{*}\left(\frac{\bar{x}^{\prime}}{\bar{x}^{*}}\right)^{\alpha} \exp \left\{\frac{\delta\left(\bar{x}^{\prime}-\bar{x}^{*}\right)}{\left(\bar{x}^{\prime}+\bar{x}^{*}\right)}\right\},
$$

The properties of the proposed estimator $t_{(\alpha, \delta)}^{(d)}$; along with cost aspects can be studied under large sample approximation, on the line of Singh et al. (2010). 


\subsection{Empirical Study}

In this section we compare the performance of different estimators considered in this paper using a population data set. The description of the population is given below.

POPULATION I. Source: Khare and Sinha (2004, p.53)

The data on physical growth of upper-socio-economic group of 95 school children of Varanasi under an ICMR study, Deportment of Pediatrics; BHU during 1983 1984 has under taken in this study. The first $25 \%$ (i.e. 24 children) units have been considered as non-response units. The values of the parameters related to the study variable $y$ (the weight in $\mathrm{Kg}$.) and the auxiliary variable $x$ (the chest circumferences in $\mathrm{cm}$.) are given below:

$$
\begin{aligned}
& \bar{Y}=19.4968, \bar{X}=55.8611, S_{y}=3.0435, S_{x}=3.2735, S_{y(2)}=2.3552, \\
& S_{x(2)}=3.5137, \rho=0.8460, \rho_{2}=0.7290, W_{2}=0.25, N=95, n=35 .
\end{aligned}
$$

We have computed the percent relative efficiency (PRE) of the proposed class of estimators $t_{(\alpha, \delta)}$ with respect to the unbiased estimator $\bar{y}^{*}$ by using the formula:

$$
\operatorname{PRE}\left(t_{(\alpha, \delta)}, \bar{y}^{*}\right)=\frac{\left(\lambda C_{y}^{2}+\theta C_{y(2)}^{2}\right)}{\left[\left(\lambda C_{y}^{2}+\theta C_{y(2)}^{2}\right)+\left(\lambda C_{x}^{2}+\theta C_{x(2)}^{2}\right)\left(\frac{(\delta+2 \alpha)^{2}}{4}-(\delta+2 \alpha) R\right)\right]} \times 100
$$

For $\alpha_{1}=0.0(0.25) 2.0, \delta_{1}=0.0(0.25) 2.0$, and $k=5(1) 2$; and findings are shown in Table 4.1. It is observed from Table 4.1 that

(i) for fixed $(\alpha, \delta)$, the PRE increases as $k$ decreases

(ii) for fixed $(\alpha \leq 1, k)$, the PRE increases as $\delta$ increases,

(iii) for fixed $(\delta, k)$, the PRE increases as $\alpha$ increases up to 1 , beyond unity no trend is observed.

For all values of $(\alpha, \delta, k)$, the PRE is larger than 100 percent which follows that the proposed class of estimators $t_{(\alpha, \delta)}$ is more efficient than the usual unbiased estimator $\bar{y}^{*}$ due to Hansen and Hurwitz (1946). For $(\alpha, \delta)=(1,0)$ in the Table 4.1 
$\operatorname{PRE}\left(t_{(\alpha, \delta)}, \bar{y}^{*}\right)$ gives the values of $\operatorname{PRE}\left(t_{R 1}, \bar{y}^{*}\right)$.

It is observed from Table 4.1 that:

(i) for and all values of $\quad \alpha \geq 1$ a $(\delta, k)$, the $\operatorname{PRE}\left(t_{(\alpha, \delta)}, \bar{y}^{*}\right) \geq 181.9835=\operatorname{PRE}\left(t_{R 1}, \bar{y}^{*}\right)$ which follows that the proposed class of estimators $t_{(\alpha, \delta)}$ is more efficient than the ratio estimator $t_{R 1}$ (for $\alpha \geq 1$ ).

(ii) (a) for $\alpha=0, k=2(1) 5$ the $\operatorname{PRE}\left(t_{(\alpha, \delta)}, \bar{y}^{*}\right)=\operatorname{PRE}\left(t_{R 1}, \bar{y}^{*}\right)$ for $\delta=2$,

(b) for $\alpha=0.25, k=2(1) 5$ the $\operatorname{PRE}\left(t_{(\alpha, \delta)}, \bar{y}^{*}\right) \geq \operatorname{PRE}\left(t_{R 1}, \bar{y}^{*}\right)$ for $\delta \geq 1.50$,

(c) for $\alpha=0.50, k=2(1) 5$ the $\operatorname{PRE}\left(t_{(\alpha, \delta)}, \bar{y}^{*}\right) \geq \operatorname{PRE}\left(t_{R 1}, \bar{y}^{*}\right)$ for $\delta \geq 1.00$,

(d) for $\alpha=0.75, k=2(1) 5$ the $\operatorname{PRE}\left(t_{(\alpha, \delta)}, \bar{y}^{*}\right) \geq \operatorname{PRE}\left(t_{R 1}, \bar{y}^{*}\right)$ for $\delta \geq 0.50$.

Thus the proposed class of estimators $t_{(\alpha, \delta)}$ is more efficient than the ratio estimator $t_{R 1}$ as long as the conditions (a) to (d) are satisfied. Larger gain in efficiency by using the proposed class of estimators $t_{(\alpha, \delta)}$ over $\bar{y}^{*}$ and $t_{R 1}$ for $1 \leq(\alpha, \delta) \leq 2$ and all the values of $k$. Finally we conclude that there is enough scope of selecting the values of scalars $(\alpha, \delta)$ involved in the class of estimators $t_{(\alpha, \delta)}$ in order to obtain estimators better than the usual unbiased estimator $\bar{y}^{*}$ and the ratio estimator $t_{R 1}$. Thus the proposal of the suggested class of estimators $t_{(\alpha, \delta)}$ is justified. For the sake of convenience to the readers, we have given the percent relative efficiencies of the proposed estimators $\bar{y}^{*}, t_{R 1}$ and $t_{i}^{*}(i=1$ to 6$)$. 


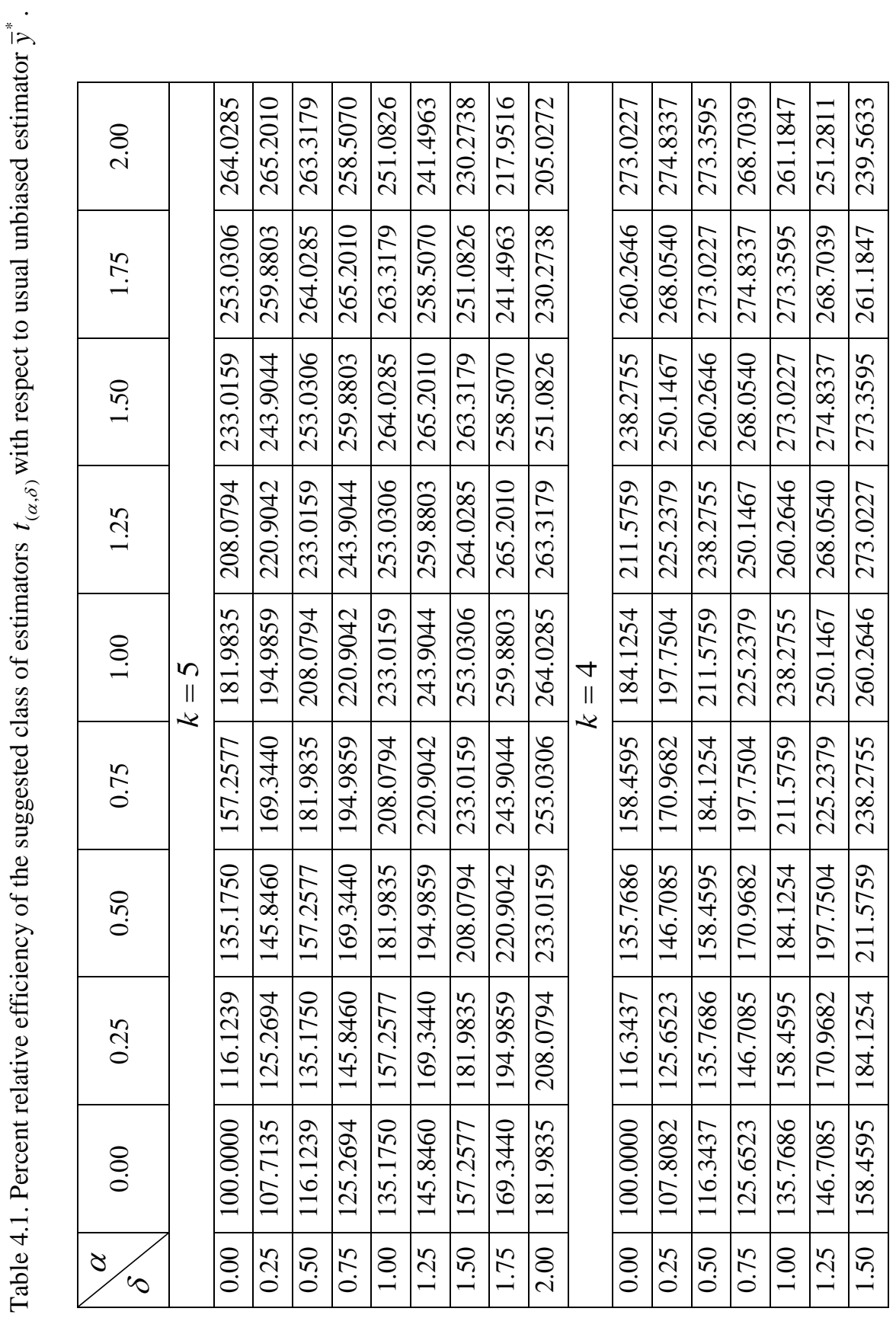




\begin{tabular}{|c|c|c|c|c|c|c|c|c|c|c|c|c|c|c|c|c|c|c|c|c|c|}
\hline 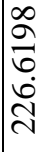 & $\begin{array}{l}\stackrel{a}{\sigma} \\
\stackrel{\alpha}{\sim}\end{array}$ & \multirow{10}{*}{$\underset{\|}{M}$} & $\begin{array}{l}1 \\
2 \\
\infty \\
\infty \\
\infty \\
\infty\end{array}$ & $\begin{array}{l}\text { నू } \\
\widehat{\sigma} \\
\infty \\
\infty \\
\infty \\
\sim\end{array}$ & 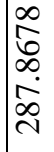 & 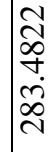 & $\begin{array}{l}2 \\
\text { ñ } \\
\infty \\
\\
\end{array}$ & 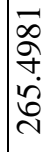 & 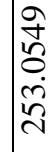 & 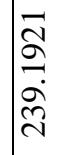 & 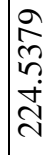 & \multirow{10}{*}{$\begin{array}{l}N \\
11 \\
N\end{array}$} & $\begin{array}{l}0 \\
0 \\
0 \\
0 \\
0 \\
0 \\
?\end{array}$ & $\begin{array}{l}\vec{\infty} \\
\stackrel{m}{m} \\
\stackrel{m}{\infty} \\
\stackrel{m}{n}\end{array}$ & $\begin{array}{l}\infty \\
\stackrel{\infty}{n} \\
\hat{\sigma} \\
\stackrel{0}{0} \\
\frac{1}{n}\end{array}$ & 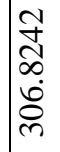 & 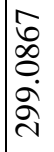 & $\begin{array}{l}\mathcal{O} \\
\delta \\
0 \\
0 \\
\infty \\
\infty \\
\infty\end{array}$ & 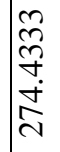 & 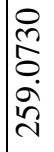 & $\begin{array}{l}\widetilde{\sigma} \\
\stackrel{N}{\sim} \\
\stackrel{\sim}{\sim}\end{array}$ \\
\hline 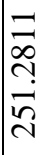 & 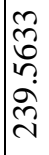 & & 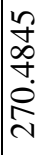 & 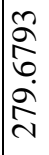 & 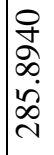 & 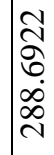 & 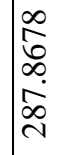 & $\begin{array}{l}\mathcal{I} \\
\infty \\
\infty \\
\dot{0} \\
\infty \\
\infty \\
\sim\end{array}$ & 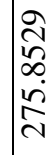 & 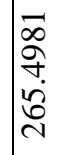 & 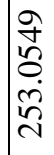 & & $\begin{array}{l}\bar{\delta} \\
\text { } \\
0 \\
\dot{0} \\
\infty \\
\text { యे }\end{array}$ & 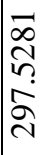 & 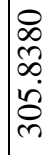 & $\begin{array}{l}\vec{\infty} \\
m \\
\tilde{m} \\
0 \\
\ddot{m}\end{array}$ & 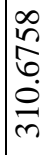 & 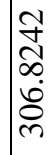 & 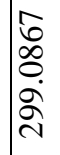 & 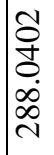 & 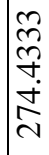 \\
\hline 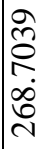 & 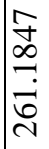 & & 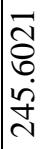 & 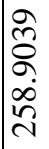 & 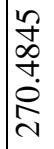 & $\begin{array}{l}2 \\
\hat{\sigma} \\
\stackrel{2}{\hat{N}}\end{array}$ & 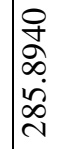 & 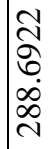 & \begin{tabular}{l}
$\infty$ \\
\multirow{\infty}{\infty}{} \\
$\infty$ \\
$\infty$ \\
$\infty$ \\
$\infty$ \\
$\sim$
\end{tabular} & \begin{tabular}{l} 
N̂ \\
$\infty$ \\
\multirow{v}{\sim}{} \\
ஸ் \\
$\infty$ \\
$\sim$
\end{tabular} & 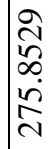 & & 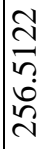 & 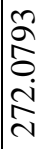 & 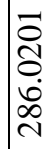 & $\begin{array}{l}\bar{\infty} \\
\stackrel{N}{N} \\
\hat{n} \\
\hat{\sim}\end{array}$ & $\begin{array}{l}\infty \\
\infty \\
\infty \\
\infty \\
\qquad \\
\mathscr{\delta} \\
\infty\end{array}$ & $\begin{array}{l}\vec{\infty} \\
m \\
\tilde{m} \\
0 \\
\tilde{m}\end{array}$ & $\begin{array}{l}\infty \\
n \\
\\
\stackrel{0}{0} \\
\mathfrak{n} \\
n\end{array}$ & 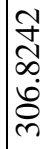 & $\begin{array}{l}\hat{0} \\
\infty \\
0 \\
\stackrel{2}{2}\end{array}$ \\
\hline 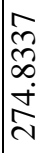 & $\begin{array}{l}n \\
\tilde{n} \\
m \\
\tilde{n} \\
\tilde{n}\end{array}$ & & 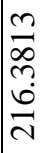 & $\begin{array}{l}\stackrel{N}{\tilde{m}} \\
\stackrel{2}{\sim} \\
\stackrel{n}{n}\end{array}$ & $\begin{array}{l}\bar{\sigma} \\
\delta \\
\delta \\
\dot{\gamma} \\
\stackrel{\sim}{d}\end{array}$ & 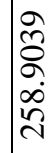 & 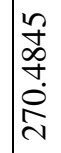 & 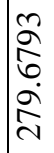 & 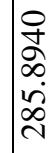 & $\begin{array}{l}\text { N } \\
\delta \\
\delta \\
\infty \\
\infty \\
\infty \\
\sim\end{array}$ & 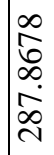 & & 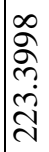 & 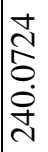 & 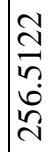 & 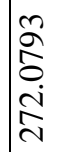 & 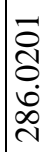 & 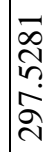 & 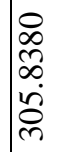 & $\begin{array}{l}\vec{\infty} \\
m \\
\tilde{m} \\
0 \\
m \\
m\end{array}$ & 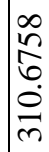 \\
\hline 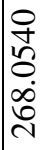 & 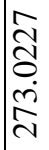 & & 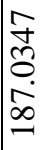 & 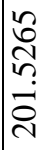 & 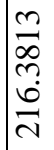 & 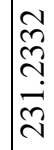 & 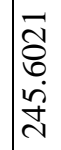 & 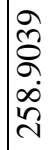 & 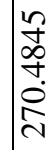 & 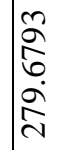 & 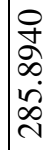 & & 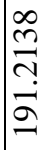 & 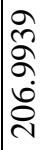 & 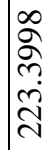 & 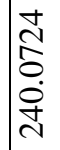 & 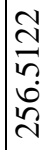 & 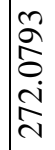 & 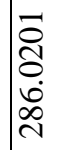 & 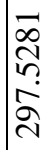 & 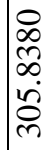 \\
\hline 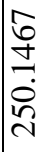 & 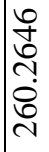 & & 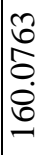 & 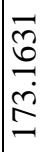 & 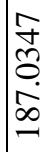 & $\begin{array}{l}\mathfrak{0} \\
\text { N } \\
\\
\tilde{\overbrace{}}\end{array}$ & 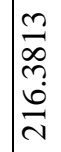 & 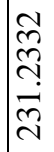 & 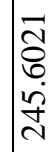 & 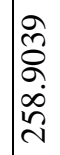 & 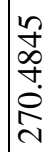 & & 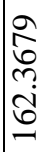 & 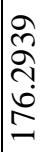 & 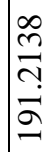 & 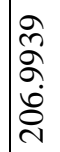 & 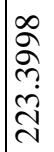 & 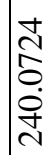 & 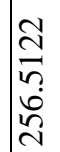 & 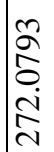 & 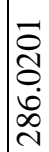 \\
\hline 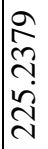 & $\begin{array}{l}n \\
\stackrel{n}{n} \\
\infty \\
\infty \\
\sim \\
\sim\end{array}$ & & 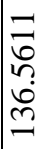 & 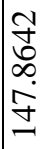 & $\begin{array}{l}0 \\
0 \\
0 \\
0 \\
8 \\
0\end{array}$ & $\begin{array}{l}\vec{\sigma} \\
\ddot{\sigma} \\
\underline{2}\end{array}$ & 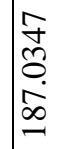 & 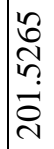 & $\begin{array}{l}m \\
\infty \\
\infty \\
\tilde{\sigma} \\
\widetilde{N}\end{array}$ & 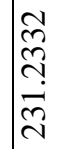 & 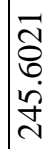 & & 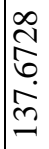 & 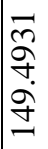 & 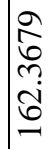 & 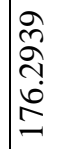 & $\begin{array}{l}\infty \\
\stackrel{\infty}{a} \\
\\
\end{array}$ & 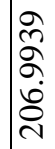 & 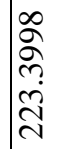 & 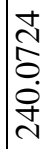 & 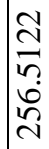 \\
\hline 吉 & 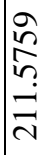 & & $\begin{array}{l}n \\
n \\
\tilde{b} \\
0 \\
=\end{array}$ & $\begin{array}{l}\vec{\sigma} \\
\stackrel{\sigma}{0} \\
\stackrel{\sim}{-}\end{array}$ & $\begin{array}{l}= \\
\overline{0} \\
n \\
0 \\
0 \\
\end{array}$ & \begin{tabular}{l}
$\mathcal{Y}$ \\
$\mathcal{J}$ \\
$\infty$ \\
\multirow{J}{J}{} \\
$\mathcal{J}$
\end{tabular} & 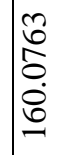 & 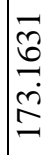 & 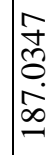 & 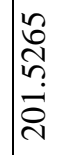 & 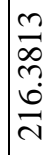 & & 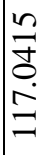 & 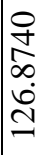 & 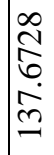 & $\begin{array}{l}\overline{\text { }} \\
\stackrel{+}{\dot{y}} \\
\dot{g}\end{array}$ & 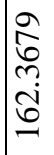 & 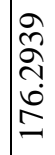 & $\begin{array}{l}\infty \\
\stackrel{\infty}{\sim} \\
\frac{\sim}{2} \\
=\end{array}$ & 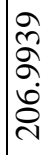 & 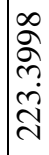 \\
\hline 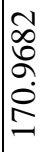 & 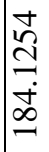 & & $\begin{array}{l}8 \\
8 \\
8 \\
8 \\
8 \\
8\end{array}$ & $\begin{array}{l}\hat{m} \\
\hat{n} \\
\hat{\sigma} \\
0\end{array}$ & $\begin{array}{l}n \\
\tilde{n} \\
\tilde{6} \\
6 \\
= \\
=\end{array}$ & $\begin{array}{l}\bar{\sigma} \\
\underset{0}{0} \\
\text { d. }\end{array}$ & 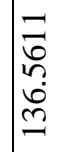 & 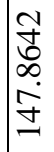 & 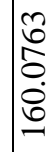 & 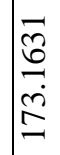 & 苍 & & $\begin{array}{l}8 \\
8 \\
8 \\
8 \\
8 \\
8\end{array}$ & $\begin{array}{l}\hat{0} \\
\stackrel{0}{\infty} \\
\stackrel{0}{0}\end{array}$ & 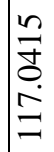 & 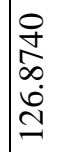 & 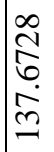 & $\begin{array}{l}\bar{n} \\
\stackrel{\alpha}{\sigma} \\
\stackrel{\sigma}{\Xi}\end{array}$ & 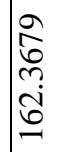 & 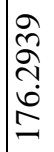 & 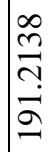 \\
\hline$\stackrel{n}{2}$ & 8 & & $\stackrel{8}{\circ}$ & ân & n̊? & $\stackrel{n}{i}$ & : & $\stackrel{\overbrace{}}{\simeq}$ & $\stackrel{n}{n}$ & $\stackrel{n}{\stackrel{2}{c}}$ & i & & $\stackrel{8}{0}$ & în & ñ & $\frac{n}{a}$ & $\stackrel{8}{8}$ & $\stackrel{2}{\check{c}}$ & $\stackrel{n}{n}$ & $\stackrel{n}{\stackrel{2}{2}}$ & $\underset{i}{8}$ \\
\hline
\end{tabular}


Table 4.2. Percent relative efficiency of the suggested class of estimators $t_{(\alpha, \delta)}$ with respect to usual unbiased estimator $\bar{y}^{*}$.

\begin{tabular}{|c|c|c|c|c|c|}
\hline \multirow{2}{*}{$(\alpha, \delta)$} & \multirow{2}{*}{ Estimator } & \multicolumn{4}{|c|}{$1 / k$} \\
\cline { 3 - 6 } & & $1 / 5$ & $1 / 4$ & $1 / 3$ & $1 / 2$ \\
\hline$(0,0)$ & $\bar{y}^{*}$ & 100.0000 & 100.0000 & 100.0000 & 100.0000 \\
\hline$(1,0)$ & $t_{R 1}$ & 181.9835 & 184.1254 & 187.0347 & 191.2138 \\
\hline$(\mathbf{2 , 0})$ & $t_{1}^{*}$ & $\mathbf{2 6 4 . 0 2 8 5}$ & $\mathbf{2 7 3 . 0 2 2 7}$ & $\mathbf{2 8 5 . 8 9 4 0}$ & $\mathbf{3 0 5 . 8 3 8 0}$ \\
\hline$(1 / 2,0)$ & $t_{2}^{*}$ & 135.1750 & 135.7686 & 136.5611 & 137.6728 \\
\hline$(0,1 / 2)$ & $t_{3}^{*}$ & 116.1239 & 116.3437 & 116.6355 & 117.4115 \\
\hline$(0,2)$ & $t_{4}^{*}$ & 181.9835 & 184.1254 & 187.0347 & 191.2138 \\
\hline$(1,1)$ & $t_{5}^{*}$ & 233.0159 & 238.2755 & 245.6021 & 256.5122 \\
\hline$(1 / 2,1 / 2)$ & $t_{6}^{*}$ & 157.2577 & 158.4595 & 160.0763 & 162.3679 \\
\hline
\end{tabular}

It is observed from Table 4.2 that ratio estimator $t_{i}^{*}$ 's ( $i=1$ to 6$)$ are more efficient than Hansen and Hurwitz (1946) estimator $\bar{y}^{*}$ which does not utilize auxiliary information. The proposed estimator $t_{4}^{*}$ is at par with the ratio estimator $t_{R 1}$. The suggested estimators $t_{1}^{*}$ and $t_{5}^{*}$ are more efficient than both the estimators $\bar{y}^{*}$ and $t_{R 1}$ with substantial gain in efficiency for all values of $(1 / k)$. Largest gain in efficiency is observed by using $t_{1}^{*}$ over $\bar{y}^{*}$.

We also note from Table 4.2 that the performance of the estimator

$$
\begin{aligned}
& t_{7}^{*}=\bar{y}^{*}\left(\frac{\bar{X}}{\bar{x}^{*}}\right) \exp \left\{\frac{2\left(\bar{X}-\bar{x}^{*}\right)}{\left(\bar{X}+\bar{x}^{*}\right)}\right\}, \\
& t_{8}^{*}=\bar{y}^{*}\left(\frac{\bar{x}^{*}}{\bar{X}}\right)^{3 / 2} \exp \left\{\frac{\bar{X}-\bar{x}^{*}}{\bar{X}+\bar{x}^{*}}\right\} ;
\end{aligned}
$$

are at par with the estimator $t_{1}^{*}$. It is also noted that the PREs of the estimators 
$t_{R 1}$ and $t_{1}^{*}$ to $t_{6}^{*}$ increase as $k$ decreases. It follows that the proposed estimator $t_{1}^{*}$ can used in practice (which do not involve any unknown constant or population parameter) in place of $\operatorname{AOE}\left(t_{(\alpha, \delta)}^{(0)}\right)$.

It is observed from Tables 4.2 and 4.5 that the PRE of the proposed estimator $t_{1}^{*}$ is near to the asymptotically optimum estimator $(\mathrm{AOE}) t_{(\alpha, \delta)}^{(0)}$. With the aid of this empirical study we conclude that the estimator that the estimators $t_{1}^{*}, t_{7}^{*}$ and $t_{8}^{*}$ appear to be appropriate choices for use in practice.

Table 4.3. Values of $\delta_{o p t}$ for different values of $(\alpha, k)$.

\begin{tabular}{|c|c|c|c|c|c|c|c|c|c|}
\hline$\alpha$ & 0.00 & 0.25 & 0.50 & 0.75 & 1.00 & 1.25 & 1.50 & 1.75 & 2.00 \\
\hline 5 & 4.2208 & 3.7208 & 3.2208 & 2.7208 & 2.2208 & 1.7208 & 1.2208 & 0.7208 & 0.2208 \\
\hline 4 & 4.2629 & 3.7629 & 3.2629 & 2.7629 & 2.2629 & 1.7629 & 1.2629 & 0.7629 & 0.2629 \\
\hline 3 & 4.3184 & 3.8184 & 3.3184 & 2.8184 & 2.3184 & 1.8184 & 1.3184 & 0.8184 & 0.3184 \\
\hline 2 & 4.3949 & 3.8949 & 3.3949 & 2.8949 & 2.3949 & 1.8949 & 1.3949 & 0.8949 & 0.3949 \\
\hline
\end{tabular}

Table 4.4. Values of $\alpha_{o p t}$ for different values of $(\delta, k)$.

\begin{tabular}{|c|c|c|c|c|c|c|c|c|c|}
\hline \multicolumn{1}{c|}{$k$} & 0.00 & 0.25 & 0.50 & 0.75 & 1.00 & 1.25 & 1.50 & 1.75 & 2.00 \\
\hline 5 & 2.1104 & 1.9854 & 1.8604 & 1.7354 & 1.6104 & 1.4854 & 1.3604 & 1.2354 & 1.1104 \\
\hline 4 & 2.1314 & 2.0064 & 1.8814 & 1.7564 & 1.6314 & 1.5064 & 1.3814 & 1.2564 & 1.1314 \\
\hline 3 & 2.1592 & 2.0342 & 1.9092 & 1.7842 & 1.6592 & 1.5342 & 1.4092 & 1.2842 & 1.1592 \\
\hline 2 & 2.1975 & 2.0725 & 1.9475 & 1.8225 & 1.6975 & 1.5725 & 1.4475 & 1.3225 & 1.1975 \\
\hline
\end{tabular}


Table 4.5. PRE of $t_{(\alpha, \delta)}$ at optimum $\left(\frac{\delta+2 \alpha}{2}\right)$ with respect to $\bar{y}^{*}$ for different values of $k$.

\begin{tabular}{|c|c|c|c|c|}
\hline$k$ & 5 & 4 & 3 & 2 \\
\hline$\left(\frac{\delta+2 \alpha}{2}\right)$ & 2.1104 & 2.1314 & 2.1592 & 2.1975 \\
\hline$P R E\left(t_{(\alpha, \delta)}^{(0)}, \bar{y}^{*}\right)$ & 265.2220 & 274.8381 & 288.8290 & 311.0505 \\
\hline
\end{tabular}

It is to be mentioned that from equation (3.17), one can calculate the optimum values of either of the constants $(\alpha, \delta)$ for different values of $k$ by fixing one of them. For the readers convenience we have given the optimum values of $(\alpha, \delta)$ in Tables 4.3 and 4.4. It is observed from Table 4.5 that the PRE of $t_{(\alpha, \delta)}$ [at optimum $\left.\left(\frac{\delta+2 \alpha}{2}\right)\right]$ with respect to $\bar{y}^{*}\left[\right.$ i.e. $\left.\operatorname{PRE}\left(t_{(\alpha, \delta)}^{(0)}, \bar{y}^{*}\right)\right]$ increases as $k$ increases.

\section{CASE II: NON-RESPONSE OCCURS ONLY ON THE STUDY VARIABLE $y$ WITH KNOWN POPULATION MEAN $\bar{X}$ OF THE AUXILIARY VARIABLE $x$}

Let the population mean $\bar{X}$ of the auxiliary variable $x$ be known. We also assume that the information on auxiliary variable $x$ is available for the complete sample size $n$. Thus in this situation we define the following class of estimators for the population mean $\bar{Y}$ as

$$
t_{\left(\alpha_{1}, \delta_{1}\right)}=\bar{y}^{*}\left(\frac{\bar{X}}{\bar{x}}\right)^{\alpha_{1}} \exp \left\{\frac{\delta_{1}(\bar{x}-\bar{X})}{(\bar{x}+\bar{X})}\right\}
$$

where $\left(\alpha_{1}, \delta_{1}\right)$ are suitable chosen constants.

To the first degree of approximation, the bias and mean squared error (MSE) of the proposed class of estimators $t_{\left(\alpha_{1}, \delta_{1}\right)}$ are respectively given by 


$$
\begin{aligned}
& B\left(t_{\left(\alpha_{1}, \delta_{1}\right)}\right)=\frac{\bar{Y}\left(\delta_{1}+2 \alpha_{1}\right)}{8} \lambda C_{x}^{2}\left(\delta_{1}+2 \alpha_{1}-4 C+2\right) \\
& \operatorname{MSE}\left(t_{\left(\alpha_{1}, \delta_{1}\right)}\right)=\bar{Y}^{2}\left[\left(\lambda C_{y}^{2}+\theta C_{y(2)}^{2}\right)+\lambda C_{x}^{2}\left\{\frac{\left(\delta_{1}+2 \alpha_{1}\right)^{2}}{4}-\left(\delta_{1}+2 \alpha_{1}\right) C\right\}\right]
\end{aligned}
$$

Equating (5.2) to zero, we have

$$
\delta_{1}=-2 \alpha_{1}\left(\text { or } \alpha_{1}=-\left(\delta_{1} / 2\right)\right)
$$

or

$$
\delta_{1}=2\left(2 C-\alpha_{1}-1\right)\left(\text { or } \alpha_{1}=2-\left(\delta_{1} / 2\right)-1\right)
$$

The proposed class of estimators $t_{\left(\alpha_{1}, \delta_{1}\right)}$ substituted with the values of $\delta_{1}$ from (5.4) and (5.5), becomes an (approximately) unbiased estimator for the population mean $\bar{Y}$.

Furthermore, if the sample size $n$ is sufficiently large, the bias of the proposed class of estimators $t_{\left(\alpha_{1}, \delta_{1}\right)}$ becomes negligible.

The MSE of $t_{\left(\alpha_{1}, \delta_{1}\right)}$ at (5.3) is minimized when

$$
\begin{aligned}
& \frac{\left(\delta_{1}+2 \alpha_{1}\right)}{2}=C, \\
& \Rightarrow\left(\delta_{1}+2 \alpha_{1}\right)=2 C .
\end{aligned}
$$

By substituting (5.6) in (5.3) we get the minimum MSE of the proposed class of estimators $t_{\left(\alpha_{1}, \delta_{1}\right)}$ as

$$
\left.\min \cdot \operatorname{MSE}\left(t_{\left(\alpha_{1}, \delta_{1}\right)}\right)=\left[\lambda S_{y}^{2}\left(1-\rho^{2}\right)+\theta S_{y(2)}^{2}\right)\right]
$$

which is the same as approximate variance of the linear regression estimator $\bar{y}_{l r}=\bar{y}^{*}+b(\bar{X}-\bar{x})$, where $b$ is the sample regression coefficient of $y$ on $x$.

Thus, we established following theorem. 
THEOREM 5.1. To the first degree of approximation,

$$
\left.\operatorname{MSE}\left(t_{\left(\alpha_{1}, \delta_{1}\right)}\right) \geq\left[\lambda S_{y}^{2}\left(1-\rho^{2}\right)+\theta S_{y(2)}^{2}\right)\right]
$$

with equality holding if

$$
\frac{\left(\delta_{1}+2 \alpha_{1}\right)}{2}=C
$$

In fact Singh and Kumar (2009) showed that the quantity $\left.\left[\lambda S_{y}^{2}\left(1-\rho^{2}\right)+\theta S_{y(2)}^{2}\right)\right]$ is the minimal possible mean squared error up to first degree of approximation for large class of estimators to which the estimator $t_{\left(\alpha_{1}, \delta_{1}\right)}$ in (5.1) also belongs, for example, for estimators of the form

$\bar{y}_{h}=\bar{y}^{*} h(\bar{x} / \bar{X})$,

where $h(\bullet)$ is a $C^{2}$-function with $h(1)=1$. Further Singh and Kumar (2009) have shown that incorporating sample and population variance of the auxiliary variable $x$ might yield an estimator that has smaller mean squared error than $\left.\left[\lambda S_{y}^{2}\left(1-\rho^{2}\right)+\theta S_{y(2)}^{2}\right)\right]$ especially when the relationship between the study variable $y$ and the auxiliary variable $x$ is markedly non-linear. Thus whatever value $C$ has, we are always able to choose an approximately optimum estimator (AOE) say $t_{\left(\alpha_{1}, \delta_{1}\right)}^{(0)}$ from the two parameter family of estimators $t_{\left(\alpha_{1}, \delta_{1}\right)}$ in (5.1).

Some members of the proposed class of estimators $t_{\left(\alpha_{1}, \delta_{1}\right)}$ of the population mean $\bar{Y} \quad$ are given in the Table 5.1 .

Table 5.1. Some members of the proposed class of estimators $t_{\left(\alpha_{1}, \delta_{1}\right)}$.

\begin{tabular}{|c|l|c|c|}
\hline S. & \multicolumn{1}{|c|}{ Estimator } & \multicolumn{2}{c|}{ Values of Constants } \\
\cline { 3 - 4 } No. & & $\alpha_{1}$ & $\delta_{1}$ \\
\hline 1. & $t_{(0,0)}=\bar{y}^{*}$ & 0 & 0 \\
\hline 2. & $t_{(1,0)}=\bar{y}^{*}(\bar{X} / \bar{x})=t_{R 2}$ & 1 & 0 \\
\hline 3. & $t_{(0,1)}=\bar{y}^{*}(\bar{x} / \bar{X})=t_{P 2}$ & -1 & 0 \\
\hline
\end{tabular}




\begin{tabular}{|c|l|c|c|}
\hline 4. & $t_{(2,0)}=\bar{y}^{*}\left(\bar{X}^{2} / \bar{x}^{2}\right)=t_{1}$ & 2 & 0 \\
\hline 5. & $t_{(1 / 2,0)}=\bar{y}^{*}(\bar{X} / \bar{x})^{1 / 2}=t_{2}$ & $1 / 2$ & 0 \\
\hline 6. & $t_{(0,1 / 2)}=\bar{y}^{*} \exp \left\{\frac{(\bar{X}-\bar{x})}{2(\bar{X}+\bar{x})}\right\}=t_{3}$ & 0 & $1 / 2$ \\
\hline 7. & $t_{(0,2)}=\bar{y}^{*} \exp \left\{\frac{2(\bar{X}-\bar{x})}{(\bar{X}+\bar{x})}\right\}=t_{4}$ & 0 & 2 \\
\hline 8. & $t_{(1,1)}=\bar{y}^{*}\left(\frac{\bar{X}}{\bar{x}}\right) \exp \left\{\frac{(\bar{X}-\bar{x})}{(\bar{X}+\bar{x})\}=t_{5}}\right.$ & 1 \\
\hline 9. & $t_{(1 / 2,1 / 2)}=\bar{y}^{*}\left(\frac{\bar{X}}{\bar{x}}\right)^{1 / 2} \exp \left\{\frac{1}{2} \frac{(\bar{X}-\bar{x})}{(\bar{X}+\bar{x})\}=t_{6}}\right.$ & $1 / 2$ & $1 / 2$ \\
\hline
\end{tabular}

To the first degree of approximation, the mean squared errors of the estimators $t_{1}$ to $t_{6}$ (listed in Table 5.1) are respectively given by

$$
\begin{aligned}
& \operatorname{MSE}\left(t_{1}\right)=\bar{Y}^{2}\left[\left(\lambda C_{y}^{2}+\theta C_{y(2)}^{2}\right)+4 C_{x}^{2}(1-C)\right], \\
& \operatorname{MSE}\left(t_{2}\right)=\bar{Y}^{2}\left[\left(\lambda C_{y}^{2}+\theta C_{y(2)}^{2}\right)+\frac{C_{x}^{2}}{4}(1-C)\right], \\
& \operatorname{MSE}\left(t_{3}\right)=\bar{Y}^{2}\left[\left(\lambda C_{y}^{2}+\theta C_{y(2)}^{2}\right)+\frac{\lambda C_{x}^{2}}{16}(1-8 C)\right], \\
& \operatorname{MSE}\left(t_{4}\right)=\bar{Y}^{2}\left[\left(\lambda C_{y}^{2}+\theta C_{y(2)}^{2}\right)+\lambda C_{x}^{2}(1-2 C)\right], \\
& \operatorname{MSE}\left(t_{5}\right)=\bar{Y}^{2}\left[\left(\lambda C_{y}^{2}+\theta C_{y(2)}^{2}\right)+3 \lambda C_{x}^{2}\left(\frac{3}{4}-C\right)\right], \\
& \operatorname{MSE}\left(t_{6}\right)=\bar{Y}^{2}\left[\left(\lambda C_{y}^{2}+\theta C_{y(2)}^{2}\right)+\frac{3 \lambda C_{x}^{2}}{2}\left(\frac{3}{8}-C\right)\right] .
\end{aligned}
$$


5.1. Comparison of Mean Squared Error of the Suggested Class of Estimators $t_{\left(\alpha_{1}, \delta_{1}\right)}$ with $\bar{y}^{*}, t_{R 2}$ and $t_{P 2}$

From (2.2) and (5.3) we have

$\operatorname{Var}\left(\bar{y}^{*}\right)-\operatorname{MSE}\left(t_{\left(\alpha_{1}, \delta_{1}\right)}\right)=\lambda C_{x}^{2} \bar{Y}^{2} \frac{\left(\delta_{1}+2 \alpha_{1}\right)}{4}\left[4 C-\left(\delta_{1}+2 \alpha_{1}\right)\right]$

which is positive if

$\left[4 C-\left(\delta_{1}+2 \alpha_{1}\right)\right]>0,\left(\delta_{1}+2 \alpha_{1}\right)>0$

i.e. if

either $\left.C>\frac{\left(\delta_{1}+2 \alpha_{1}\right)}{4},\left(\delta_{1}+2 \alpha_{1}\right)>0\right)$.

or $\left.\quad C<\frac{\left(\delta_{1}+2 \alpha_{1}\right)}{4},\left(\delta_{1}+2 \alpha_{1}\right)<0\right\}$

From (2.9) and (5.3) we have

$\operatorname{MSE}\left(t_{R 2}\right)-\operatorname{MSE}\left(t_{\left(\alpha_{1}, \delta_{1}\right)}\right)=\lambda C_{x}^{2} \bar{Y}^{2}\left\{1-\frac{\left(\delta_{1}+2 \alpha_{1}\right)}{2}\right\}\left[1+\frac{\left(\delta_{1}+2 \alpha_{1}\right)}{2}-2 C\right]$

which is non-negative if

$\left.\begin{array}{l}\text { either } C>\frac{\left(\delta_{1}+2 \alpha_{1}+2\right)}{4}, \frac{\left(\delta_{1}+2 \alpha_{1}\right)}{2}>1 \\ \text { or } \quad C<\frac{\left(\delta_{1}+2 \alpha_{1}+2\right)}{4}, \frac{\left(\delta_{1}+2 \alpha_{1}\right)}{2}<1\end{array}\right\}$.

Further, from (2.11) and (5.3) we have

$\operatorname{MSE}\left(t_{P 2}\right)-\operatorname{MSE}\left(t_{\left(\alpha_{1}, \delta_{1}\right)}\right)=\lambda C_{x}^{2} \bar{Y}^{2}\left\{1+\frac{\left(\delta_{1}+2 \alpha_{1}\right)}{2}\right\}\left[2 C-\frac{\left(\delta_{1}+2 \alpha_{1}\right)}{2}+1\right]$

which is greater than zero if

either $C>-\frac{1}{2}+\frac{\left(\delta_{1}+2 \alpha_{1}\right)}{4}, \frac{\left(\delta_{1}+2 \alpha_{1}\right)}{2}>-1$.

or $\left.\quad C<-\frac{1}{2}+\frac{\left(\delta_{1}+2 \alpha_{1}\right)}{4}, \frac{\left(\delta_{1}+2 \alpha_{1}\right)}{2}<-1\right\}$

Thus it follows that the proposed class of estimators $t_{\left(\alpha_{1}, \delta_{1}\right)}$ is more efficient than the usual unbiased estimator $\bar{y}^{*}$, ratio estimator $t_{R 2}$ and product estimator $t_{P 2}$ as long as the conditions in (5.16), (5.17) and (5.18) respectively hold true. 
5.2. Comparison of the proposed estimator $t_{j}(j=1$ to 6$)$ with respect to usual unbiased estimator $\bar{y}^{*}$ and the ratio estimator $t_{R 2}$

It can be shown that the suggested class of estimators:

(i) $t_{1}$ is more efficient than $\bar{y}^{*}$ and $t_{R 2}$ respectively if

$$
C>1
$$

and

$$
C>\frac{3}{2}
$$

(ii) $t_{2}$ is more efficient than $\bar{y}^{*}$ and $t_{R 2}$ respectively if

$$
C>\frac{1}{4}
$$

and

$$
C>\frac{3}{4}
$$

(iii) $t_{3}$ is more efficient than $\bar{y}^{*}$ and $t_{R 2}$ respectively if

$$
C>\frac{1}{8}
$$

and

$$
C>\frac{5}{8}
$$

(iv) $t_{4}$ is more efficient than $\bar{y}^{*}$ and $t_{R 2}$ respectively if

$$
C>\frac{1}{2}
$$

and

$$
C>1 \text {. }
$$

(v) $t_{5}$ is more efficient than $\bar{y}^{*}$ and $t_{R 2}$ respectively if

$$
C>\frac{3}{4}
$$

and 


$$
C>\frac{5}{4}
$$

(vi) $t_{6}$ is more efficient than $\bar{y}^{*}$ and $t_{R 2}$ respectively if

$$
C>\frac{3}{8}
$$

and

$$
C>\frac{7}{8}
$$

REMARK 5.2.1. Following the same procedure as adopted by Rao (1983), the cost aspects can be also studied when there is non-response only on the variable $y$.

REMARK 5.2.2. The double sampling version of suggested class of estimators $t_{\left(\alpha_{1}, \delta_{1}\right)}$ given by (5.1) can be given when the population mean $\bar{X}$ is not known. Suppose that complete information on the auxiliary variable $x$ is available for both the first and second samples, and that incomplete information on the study variable $y$ is available.

So, in this case, we use information on the $\left(n_{1}+r\right)$ responding units on the study variable $y$, and complete information on the auxiliary variable $x$ from the sample of size $n$. Thus one can suggest a double sampling version of the class of estimators $t_{\left(\alpha_{1}, \delta_{1}\right)}$ defined at (5.1) for population mean $\bar{Y}$ when the non-response occurs only on the study variable $y$ as

$$
t_{\left(\alpha_{1}, \delta_{1}\right)}^{(d)}=\bar{y}^{*}\left(\frac{\bar{x}^{\prime}}{\bar{x}}\right)^{\alpha_{1}} \exp \left\{\frac{\delta_{1}\left(\bar{x}-\bar{x}^{\prime}\right)}{\left(\bar{x}+\bar{x}^{\prime}\right)}\right\},
$$

The properties of the suggested class of estimators $t_{\left(\alpha_{1}, \delta_{1}\right)}^{(d)}$ along with cost aspects can be studied under large sample approximation, on the line of Tabasum and Khan (2006) and Singh et al.(2011). 


\subsection{Empirical Study}

In this section, we consider the same population data set which is given in Section 4.3. We have computed the percent relative efficiency (PRE) of the proposed class of estimators $t_{\left(\alpha_{1}, \delta_{1}\right)}$ with respect the usual unbiased estimator $\bar{y}^{*}$ by using the formula:

$$
\operatorname{PRE}\left(t_{\left(\alpha_{1, \delta_{1}}\right)}, \bar{y}^{*}\right)=\frac{\left(\lambda C_{y}^{2}+\theta C_{y(2)}^{2}\right)}{\left[\left(\lambda C_{y}^{2}+\theta C_{y(2)}^{2}\right)+\lambda C_{x}^{2}\left(\frac{\left(\delta_{1}+2 \alpha_{1}\right)^{2}}{4}-\left(\delta_{1}+2 \alpha_{1}\right) C\right)\right]} \times 100 .
$$

For $\alpha_{1}=0.0(0.25) 2.0, \delta_{1}=0.0(0.25) 2.0$, and $k=5(1) 2$; and findings are shown in Table 5.2.

Table 5.2 exhibits that the values of PREs are larger than 100 percent. It follows that the proposed class of estimators $t_{\left(\alpha_{1}, \delta_{1}\right)}$ is better than usual unbiased estimator $\bar{y}^{*}$ for the values of the constants $\left(\alpha_{1}, \delta_{1}\right)$ and $k$ considered here. It is further observed that the proposed class of estimators $t_{\left(\alpha_{1}, \delta_{1}\right)}$ is better than the ratio estimator $t_{R 2}$ for $1 \leq \alpha_{1}, \delta_{1} \leq 2$ and $k=5(1) 2$. Comparing the Tables 4.1 and 5.2 we find that the $\operatorname{PRE}\left(t_{(\alpha, \delta)}, \bar{y}^{*}\right)$ [i.e. when there is non-response present in both the variables $y$ and $x$ ] is larger than the class of estimators $t_{\left(\alpha_{1}, \delta_{1}\right)}$ [i.e. when there is non-response occurs only on the study variables $y$ and information on the auxiliary variable $x$ is available for complete sample size $n$ ]. 


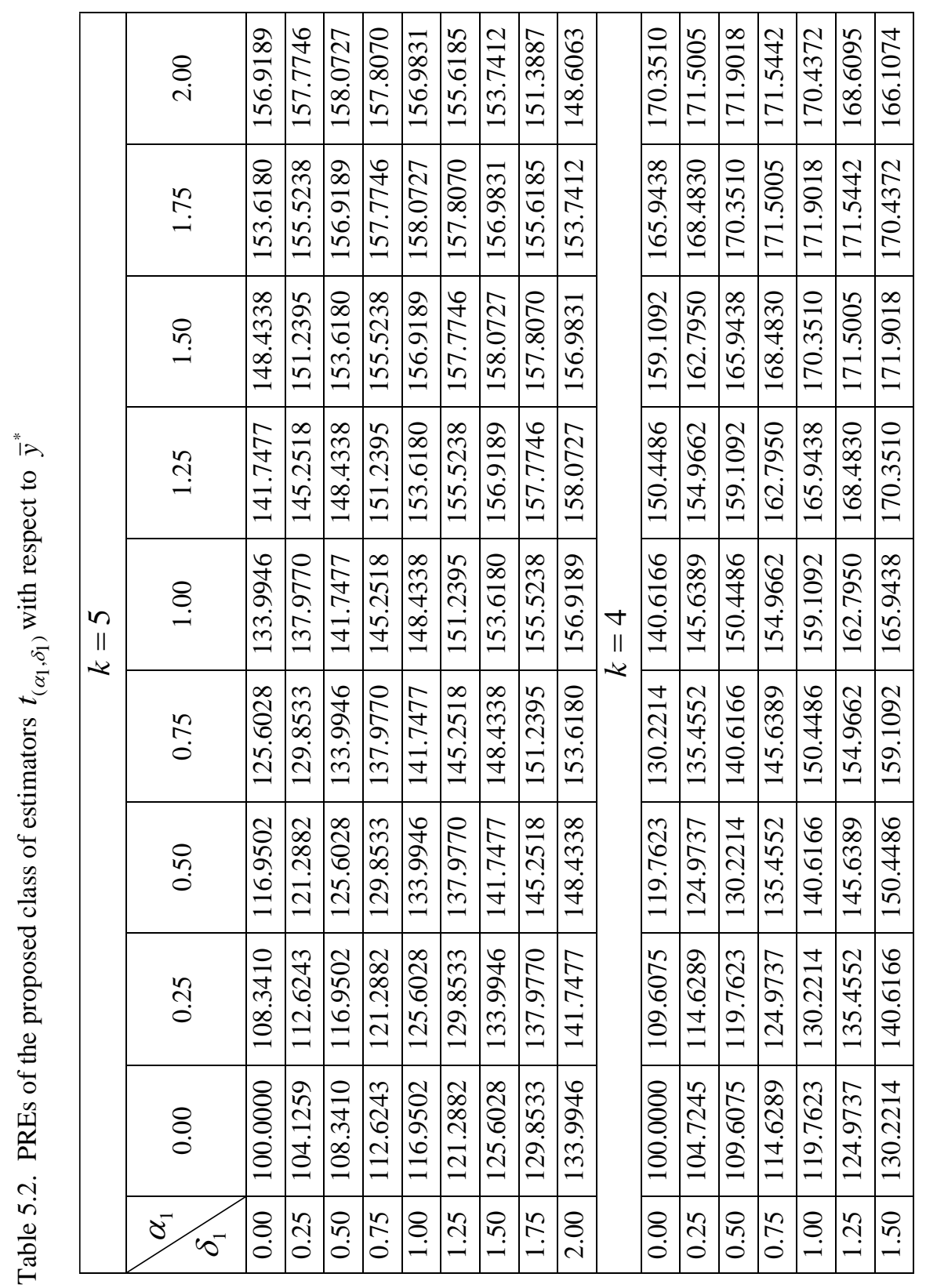




\begin{tabular}{|c|c|c|c|c|c|c|c|c|c|c|c|c|c|c|c|c|c|c|c|c|c|}
\hline 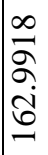 & $\hat{a}$ & & 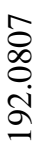 & $\begin{array}{l}\infty \\
\dot{\alpha} \\
\tilde{\sigma}\end{array}$ & 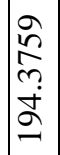 & $\begin{array}{l}n \\
\tilde{n} \\
\infty \\
\dot{2} \\
\tilde{\sigma}\end{array}$ & 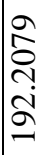 & $\begin{array}{l}\infty \\
\infty \\
\curvearrowright \\
\infty\end{array}$ & $\begin{array}{l}\hat{n} \\
\infty \\
\infty \\
\infty \\
\infty \\
\infty\end{array}$ & 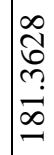 & 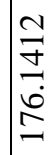 & & & 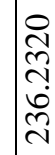 & 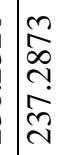 & 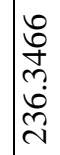 & 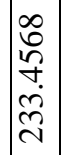 & $\mid \begin{array}{l}\mathscr{n} \\
\infty \\
\sim \\
\sim \\
\infty \\
\sim \\
\sim\end{array}$ & 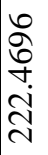 & 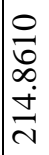 & 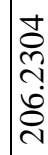 \\
\hline $\mid \begin{array}{l}2 \\
8 \\
8 \\
\infty \\
\infty \\
0\end{array}$ & : & & 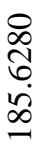 & $\begin{array}{l}\tilde{N} \\
\tilde{m} \\
\tilde{\infty} \\
\infty\end{array}$ & 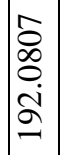 & $\begin{array}{l}\infty \\
\infty \\
\infty \\
\stackrel{\infty}{r} \\
\stackrel{2}{=}\end{array}$ & \begin{tabular}{l}
$\stackrel{a}{n}$ \\
$\hat{n}$ \\
\multirow{2}{*}{} \\
$=$
\end{tabular} & $\begin{array}{l}n \\
\tilde{n} \\
\infty \\
\dot{2} \\
2 \\
2\end{array}$ & 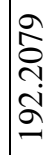 & $\begin{array}{l}\infty \\
\infty \\
\infty \\
\infty \\
\infty\end{array}$ & $\begin{array}{l}n \\
\tilde{n} \\
\infty \\
\infty \\
\infty \\
\infty\end{array}$ & & $\begin{array}{l}\mathcal{B} \\
\text { ju } \\
\text { v }\end{array}$ & 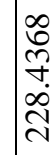 & $\begin{array}{l}m \\
\tilde{n} \\
\tilde{m} \\
\tilde{n}\end{array}$ & 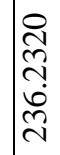 & 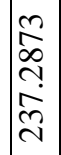 & 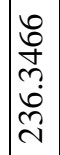 & 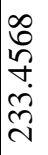 & 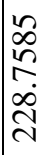 & 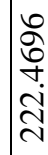 \\
\hline 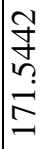 & 7 & & & $\begin{array}{l}8 \\
\infty \\
\infty \\
\infty \\
\infty \\
\infty\end{array}$ & $\begin{array}{l}\infty \\
\widetilde{\sigma} \\
\dot{\sim} \\
\infty\end{array}$ & 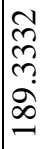 & 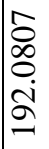 & 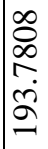 & $\begin{array}{l}\stackrel{2}{n} \\
\\
\dot{a} \\
2\end{array}$ & $\begin{array}{l}n \\
n \\
\infty \\
\tilde{a} \\
\tilde{2}\end{array}$ & 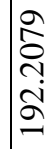 & & $\dot{s}$ & 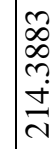 & : & 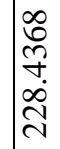 & $\begin{array}{l}\tilde{m} \\
\tilde{n} \\
\tilde{m} \\
\tilde{n}\end{array}$ & 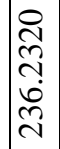 & 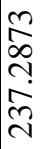 & 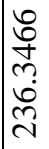 & 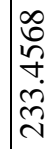 \\
\hline 每 & 17 & & 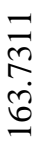 & $\begin{array}{l}\frac{n}{2} \\
\hat{\sigma} \\
\hat{\sigma}\end{array}$ & $\begin{array}{l}\stackrel{ }{\Xi} \\
\infty \\
\stackrel{\sim}{\Sigma} \\
\end{array}$ & 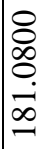 & $\begin{array}{l}\infty \\
\infty \\
\text { ర్ర } \\
\infty \\
\infty \\
\infty\end{array}$ & $\begin{array}{l}\tilde{N} \\
\tilde{m} \\
\tilde{\infty} \\
\infty \\
\infty\end{array}$ & 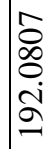 & $\begin{array}{l}\infty \\
\infty \\
\infty \\
\stackrel{\infty}{\sigma} \\
\stackrel{2}{2}\end{array}$ & $a$ & & $\begin{array}{l}n \\
\text { م } \\
\\
\infty \\
\infty\end{array}$ & 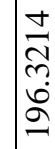 & 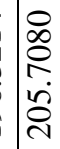 & $\begin{array}{l}n \\
\infty \\
\infty \\
\tilde{N} \\
\dot{J} \\
\tilde{N}\end{array}$ & 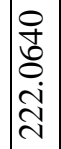 & 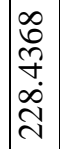 & $\begin{array}{l}m \\
\tilde{n} \\
\\
\tilde{n} \\
\tilde{n}\end{array}$ & 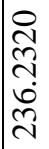 & 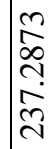 \\
\hline \begin{tabular}{l}
0 \\
$\infty$ \\
$\infty$ \\
\multirow{\infty}{\infty}{} \\
$\infty$ \\
$\infty$ \\
0
\end{tabular} & ñ & $m$ & 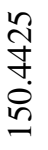 & 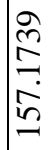 & $\begin{array}{l}\stackrel{m}{r} \\
\tilde{\vartheta}\end{array}$ & $\begin{array}{l}\frac{n}{\sigma} \\
\hat{\sigma} \\
\underline{\sigma}\end{array}$ & 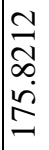 & 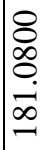 & $\begin{array}{l}\stackrel{0}{\infty} \\
\mathbb{U} \\
\ddot{\infty} \\
\infty \\
0\end{array}$ & $\begin{array}{l}\tilde{N} \\
\tilde{\infty} \\
\tilde{\infty} \\
\infty\end{array}$ & 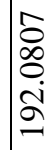 & & 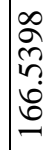 & 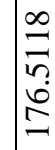 & 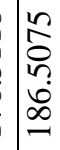 & 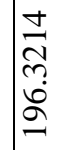 & 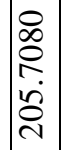 & $\begin{array}{l}\tilde{\infty} \\
\infty \\
\tilde{m} \\
\dot{\Xi} \\
\widetilde{N}\end{array}$ & 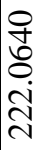 & 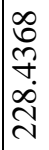 & $\begin{array}{l}\tilde{m} \\
\tilde{\sim} \\
\tilde{m} \\
\tilde{n}\end{array}$ \\
\hline 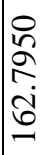 & $\stackrel{m}{\mathscr{Z}}$ & & $\begin{array}{l}\vec{\curvearrowright} \\
\stackrel{\infty}{\infty} \\
\dot{0}\end{array}$ & $\begin{array}{l}\stackrel{\partial}{+} \\
\dot{\Xi} \\
\dot{\theta} \\
\dot{\Xi}\end{array}$ & 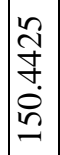 & $\frac{\partial}{\stackrel{2}{n}}$ & $\begin{array}{l}= \\
\stackrel{n}{\sim} \\
\stackrel{\infty}{\sigma}\end{array}$ & $\begin{array}{l}n \\
\hat{\sigma} \\
\hat{\sigma} \\
\hat{\sigma}\end{array}$ & 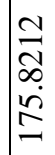 & $\begin{array}{l}8 \\
\infty \\
\infty \\
\dot{\infty} \\
\infty \\
-\end{array}$ & 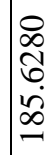 & & 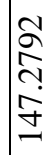 & 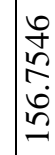 & $\begin{array}{l}\infty \\
\infty \\
\tilde{n} \\
\sim \\
0 \\
0\end{array}$ & 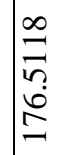 & $\begin{array}{l}n \\
\tilde{o} \\
0 \\
n \\
\infty \\
\infty \\
-1\end{array}$ & 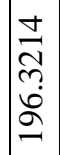 & 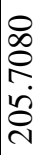 & 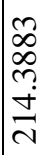 & ㄱ. \\
\hline 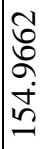 & $?$ & & $\begin{array}{l}\bar{\Omega} \\
\text { ğ } \\
\text { ஸ் }\end{array}$ & 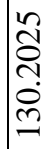 & 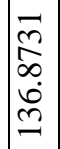 & 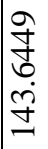 & 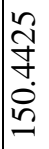 & $\begin{array}{l}\stackrel{2}{2} \\
\stackrel{2}{n} \\
i n\end{array}$ & $\begin{array}{l}= \\
\approx \\
\stackrel{\vec{n}}{0} \\
\underline{\sigma}\end{array}$ & $\begin{array}{l}\frac{n}{2} \\
\hat{\sigma} \\
\text { g}\end{array}$ & $\stackrel{n}{\Sigma}$ & & $\begin{array}{l}\text { 方 } \\
\text { ñ } \\
\text { ปิ }\end{array}$ & 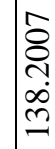 & 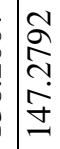 & 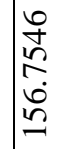 & 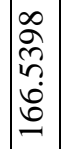 & $\begin{array}{l}\infty \\
\bar{n} \\
\tilde{v} \\
\simeq\end{array}$ & 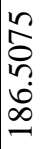 & 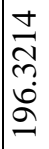 & ஜֶ. \\
\hline 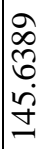 & - & & $\nabla$ & 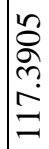 & $\left|\begin{array}{l}\hat{\Omega} \\
\dot{\theta}\end{array}\right|$ & 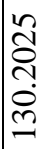 & 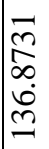 & 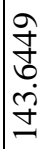 & 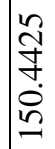 & 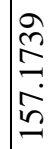 & $\underline{6}$ & & $\begin{array}{l}\frac{n}{2} \\
\frac{1}{2} \\
\text { m. } \\
=\end{array}$ & 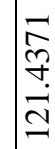 & $\begin{array}{l}\tilde{n} \\
\tilde{n} \\
\tilde{n} \\
\mathfrak{n}\end{array}$ & $\begin{array}{l}\hat{\sigma} \\
\delta \\
\text { N̦ } \\
\infty \\
\end{array}$ & 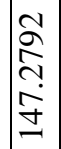 & \begin{tabular}{l}
0 \\
\multirow{1}{n}{} \\
$r$ \\
0 \\
$n$ \\
$n$
\end{tabular} & \begin{tabular}{l}
$\infty$ \\
$\Omega$ \\
\\
\\
\hdashline \\
0
\end{tabular} & 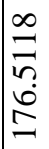 & $\begin{array}{l}0 \\
\mathscr{n} \\
\infty \\
\infty\end{array}$ \\
\hline \begin{tabular}{l}
$\sqrt{n}$ \\
$\tilde{n}$ \\
\multirow{2}{n}{} \\
$\stackrel{n}{2}$ \\
\end{tabular} & 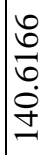 & & \& & 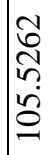 & 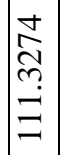 & 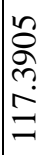 & $\begin{array}{l}\bar{\sigma} \\
\hat{b} \\
\stackrel{d}{c}\end{array}$ & 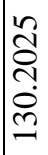 & $\begin{array}{l}\bar{m} \\
\infty \\
\dot{\infty} \\
\infty\end{array}$ & 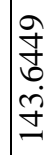 & $\mathrm{N}$ & & ஓ & 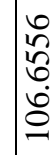 & $\left\{\begin{array}{l}n \\
\frac{2}{2} \\
\frac{2}{2}\end{array}\right.$ & $\begin{array}{l}\bar{N} \\
\stackrel{m}{\vartheta} \\
\stackrel{\sim}{\beth}\end{array}$ & $\begin{array}{l}a \\
\tilde{n} \\
\tilde{n} \\
\mathfrak{\beth} \\
\end{array}$ & 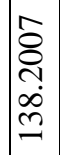 & 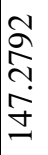 & 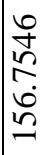 & ñ \\
\hline 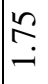 & $1^{\circ}$ & & & a & ^ & $\stackrel{n}{r}$ & $\underset{8}{8}$ & $\stackrel{\overbrace{}}{\sim}$ & $\stackrel{n}{n}$ & $\stackrel{n}{r}$ & & & & s. & 유 & $\stackrel{n}{\stackrel{0}{0}}$ & $\underset{-}{8}$ & $\stackrel{n}{\sim}$ & $\stackrel{n}{-}$ & $\stackrel{n}{\stackrel{2}{*}}$ & 0 \\
\hline
\end{tabular}


We have also given the percent relative efficiency of different estimators $t_{R 2}$ and $t_{1}$ to $t_{6}$ with respect to usual unbiased estimator $\bar{y}^{*}$ for different values of $k=5(1) 2$ in Table 5.3.

Table 5.3. Percent relative efficiency of the suggested class of estimators $t_{(\alpha, \delta)}$ with respect to usual unbiased estimator $\bar{y}^{*}$.

\begin{tabular}{|c|c|c|c|c|c|c|}
\hline \multirow{2}{*}{$\alpha_{1}$} & \multirow{2}{*}{$\delta_{1}$} & \multirow{2}{*}{ Estimator } & \multicolumn{5}{|c|}{$1 / k$} \\
\cline { 4 - 7 } & & & $1 / 5$ & $1 / 4$ & $1 / 3$ & $1 / 2$ \\
\hline 0 & 0 & $\bar{y}^{*}$ & 100.0000 & 100.0000 & 100.0000 & 100.0000 \\
\hline 1 & 0 & $t_{R 2}$ & 133.9946 & 140.6166 & 150.4425 & 166.5398 \\
\hline $\mathbf{2}$ & $\mathbf{0}$ & $t_{1}$ & $\mathbf{1 5 6 . 9 1 8 9}$ & $\mathbf{1 7 0 . 3 5 1 0}$ & $\mathbf{1 9 2 . 6 2 8 0}$ & $\mathbf{2 3 3 . 2 3 3 3}$ \\
\hline $1 / 2$ & 0 & $t_{2}$ & 116.9502 & 119.7623 & 123.6931 & 129.5759 \\
\hline 0 & $1 / 2$ & $t_{3}$ & 108.3410 & 109.6075 & 111.3274 & 113.7975 \\
\hline 0 & 2 & $t_{4}$ & 133.9946 & 140.6166 & 150.4425 & 166.5398 \\
\hline 1 & 1 & $t_{5}$ & 148.4338 & 159.1092 & 175.8212 & 205.7080 \\
\hline $1 / 2$ & $1 / 2$ & $t_{6}$ & 125.6028 & 130.2214 & 136.8731 & 147.2792 \\
\hline
\end{tabular}

We have further computed the optimum values of $\alpha_{1}$ for given values of $\delta_{1}$, and optimum values of $\delta_{1}$ for given of $\alpha_{1}$ respectively tabulated in Tables 5.4 and 5.5.

Table 5.4. Values of $\delta_{1 \text { opt }}$ for different values of $\left(\alpha_{1}, k\right)$.

\begin{tabular}{|c|c|c|c|c|c|c|c|c|c|}
\hline$\alpha_{1}$ & 0.00 & 0.25 & 0.50 & 0.75 & 1.00 & 1.25 & 1.50 & 1.75 & 2.00 \\
\hline 5 & 4.5072 & 4.2572 & 4.0072 & 3.7572 & 3.5072 & 3.2572 & 3.0072 & 2.7572 & 2.5072 \\
\hline 4 & 4.5072 & 4.2572 & 4.0072 & 3.7572 & 3.5072 & 3.2572 & 3.0072 & 2.7572 & 2.5072 \\
\hline 3 & 4.5072 & 4.2572 & 4.0072 & 3.7572 & 3.5072 & 3.2572 & 3.0072 & 2.7572 & 2.5072 \\
\hline 2 & 4.5072 & 4.2572 & 4.0072 & 3.7572 & 3.5072 & 3.2572 & 3.0072 & 2.7572 & 2.5072 \\
\hline
\end{tabular}


Table 5.5. Values of $\alpha_{1 \text { opt }}$ for different values of $\left(\delta_{1}, k\right)$.

\begin{tabular}{|c|c|c|c|c|c|c|c|c|c|}
\hline 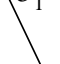 & 0.00 & 0.25 & 0.50 & 0.75 & 1.00 & 1.25 & 1.50 & 1.75 & 2.00 \\
\hline 5 & 4.5072 & 4.0072 & 3.5072 & 3.0072 & 2.5072 & 2.0072 & 1.5072 & 1.0072 & 0.5072 \\
\hline 4 & 4.5072 & 4.0072 & 3.5072 & 3.0072 & 2.5072 & 2.0072 & 1.5072 & 1.0072 & 0.5072 \\
\hline 3 & 4.5072 & 4.0072 & 3.5072 & 3.0072 & 2.5072 & 2.0072 & 1.5072 & 1.0072 & 0.5072 \\
\hline 2 & 4.5072 & 4.0072 & 3.5072 & 3.0072 & 2.5072 & 2.0072 & 1.5072 & 1.0072 & 0.5072 \\
\hline
\end{tabular}

Table 5.6. PRE of $t_{\left(\alpha_{1}, \delta_{1}\right)}$ at optimum $\left(\frac{\delta_{1}+2 \alpha_{1}}{2}\right)$ with respect to $\bar{y}^{*}$ for different values of $k$.

\begin{tabular}{|c|c|c|c|c|}
\hline$k$ & 5 & 4 & 3 & 2 \\
\hline$\left(\frac{\delta_{1}+2 \alpha_{1}}{2}\right)_{o p t}$ & 2.2536 & 2.2536 & 2.2536 & 2.2536 \\
\hline $\operatorname{PRE}\left(t_{\left(\alpha_{1}, \delta_{1}\right)}^{(0)}, \bar{y}^{*}\right)$ & 158.0729 & 171.9021 & 194.3764 & 237.2882 \\
\hline
\end{tabular}

Tables 5.3 and 5.6 demonstrate that the proposed estimator $t_{1}$ gives the PRE closer to the asymptotically optimum estimator (AOE) $t_{\left(\alpha_{1}, \delta_{1}\right)}^{(0)}$. Thus in practice $t_{1}$ is to be preferred as an alternative to AOE. In general, there is increasing trend in PRE as $k$ decreases. The PRE of $t_{5}$ with respect to $\bar{y}^{*}$ is larger than that of $t_{R 2}, t_{2}, t_{3}, t_{6}$. Also $\operatorname{PRE}\left(t_{4}, \bar{y}^{*}\right)$ is at par with $\operatorname{PRE}\left(t_{R 2}, \bar{y}^{*}\right)$. It follows that the estimator $t_{1}$ and $t_{5}$ are appropriate choice among the estimators $\bar{y}^{*}, t_{R 2}, t_{1}, t_{2}, t_{3}, t_{4} t_{5}$ and $t_{6}$. Comparing Table 5.2 and Table 5.3 we conclude that there is enough scope of selecting the values of scalars $\left(\alpha_{1}, \delta_{1}\right)$ in order to obtain estimators better than usual unbiased estimator $\bar{y}^{*}$ and the ratio estimator $t_{R 2}$ from the proposed class of estimators $t_{\left(\alpha_{1}, \delta_{1}\right)}$. Thus our recommendation is in the favor of proposed class of 
estimators $t_{\left(\alpha_{1}, \delta_{1}\right)}$ regarding its use in practice.

Finally, it is observed that the estimator formulated in Case I [i.e. when there is nonresponse present on both the variables $y$ and $x$ ] is more efficient than the corresponding estimators in Case II [i.e. when there is non-response occurs only on the study variables $y$ ].

\section{REFERENCES}

Chanu, W.W. and Singh, B.K. (2015): Improved exponential ratio-cum-exponential dual to ratio estimator of finite population mean in presence of non-response. Jour. Appl. Prob., 4(1), 103-111. Cochran, W. G. (1977): Sampling Techniques. $3^{\text {rd }}$ edn. New York, John Wiley and Sons.

Hansen, M. H. and Hurwitz, W.N. (1946): The problem of non-response in sample surveys. Jour. Amer. Statist. Assoc., 41, 517-529.

Kadilar, C. and Cingi, H. (2003): A study on the chain ratio-type estimator. Hacett. Jour. Math. Statist., 32(1), 105-108.

Khare, B.B. and Sinha, R. R. (2004): Estimation of finite population ratio using two phase sampling scheme in presence of non-response. Aligarh Jour. Statist., 24, 43-56.

Khare, B.B. and Srivastava, S. (1997): Transformed ratio type estimators for the population mean in the presence of non-response. Commun. Statist. Theo. Meth., 26(7), 1779-1791.

Kumar, S. (2012): Ratio-cum-regression estimator for estimating population mean with sub-sampling of non- respondents. Communications of the Korean Statist. Soci., 19(5), 663-672.

Kumar, S. and Vishwanathaiah, M. (2013): A generalized family of transformed estimators in the presence of non-response in sample surveys. Jour. Adv. Comp., 2(3), 99-109.

Kumar, S., Singh, H.P., Bhougal, S. and Gupta, R. (2011): A class of ratio-cum-product type estimators under double sampling in presence of non-response. Hacett. Jour. Math. Statist., 40(4), 589-599.

Olufadi, Y. and Kumar, S. (2014): Ratio-cum-product estimator using exponential estimator in the presence of non response. Jour. Adv. Comp., 3(1), 1-11.

Rao, P.S.R.S. (1986): Ratio estimation with sub-sampling the non-respondents. Survey. Method., 12(2), 217-230.

Singh, H. P. and Kumar, S. (2008): A regression Approach to the estimation of the finite population mean in the presence of non-response. Aust. N. Z. Jour. Stat., 50(4), 395-408.

Singh, H. P. and Kumar, S. (2009): A general class of estimators of the population mean in survey sampling using auxiliary information with sub sampling the non- respondents. The Korean Jour. Appl. Statist., 22(2), 387-402.

Singh, H.P. and Pal, S.K. (2015): A new chain ratio-ratio-type exponential estimator using auxiliary information in sample surveys. Int. Jour. Maths and its Appl., 3(4-B), 37-46.

Singh, H.P., Kumar, S. and Kozak, M. (2010): Improved estimation of finite population mean using subsampling to deal with non-response in two phase sampling scheme. Commun. Statist. Theo. Meth., 39, 112.

Srivastava, S.K. (1971): A generalized estimator using multi-auxiliary information. Jour. Amer. Statist. Associ., 66(334), 404-407.

Srivastava, S.K. (1990): A class of estimator using auxiliary information in sample surveys. The Canadian Jour. Statist., 8(2), 253-254. 
Swain, A.K. P.C. (2014): On an improved ratio type estimator of finite population in sample surveys, Revista. Invest. Opera., 35(1), 49-57.

Tabasum, R. and Khan, I.A. (2006): Double sampling ratio estimator for the population mean in presence of non-response. Assam Statist. Rev., 20(1), 73-83.

\section{Surya K. Pal}

School of Studies in Statistics,

Vikram University, Ujjain-456010, M.P., India email: suryakantpal6676@gmail.com

\section{Housila P. Singh}

School of Studies in Statistics,

Vikram University, Ujjain-456010, M.P., India 